\title{
FREQUENCIES OF SINGLE-NUCLEOTIDE POLYMORPHISMS AND HAPLOTYPES OF THE SLCO1B1 GENE IN SELECTED POPULATIONS OF THE WESTERN BALKANS
}

\author{
Daka Grapci A ${ }^{1}$, Dimovski AJ ${ }^{2}$, Kapedanovska A ${ }^{2}$, Vavlukis $\mathrm{M}^{3}$, Eftimov A ${ }^{2}$, Matevska
} Geshkovska N², Labachevski N ${ }^{4}$, Jakjovski $\mathrm{K}^{4}$, Gorani $\mathrm{D}^{5}$, Kedev $\mathrm{S}^{3}$, Mladenovska K,*

\begin{abstract}
*Corresponding Author: Professor Kristina Mladenovska, Faculty of Pharmacy, Center for Biomolecular Pharmaceutical Analyses, University "Ss Cyril and Methodius" in Skopje, Blv. "Mother Theresa" 47, 1000 Skopje, Republic of Macedonia. Tel: +389-2-3126-032. Fax: +389-2-3132-015. E-mail: krml@ff.ukim.edu.mk
\end{abstract}

\begin{abstract}
As a membrane influx transporter, organic anion-transporting polypeptide 1B1 (OATP1B1) regulates the cellular uptake of a number of endogenous compounds and drugs. The aim of this study was to characterize the diversity of the solute carrier organic anion transporter family member 1B1 (SLCO1B1) gene encoding this transporter in two ethnic groups populating the Western Balkans. The distribution of SCLO1B1 alleles was determined at seven variant sites (c. $388 \mathrm{~A}>\mathrm{G}$, c. $521 \mathrm{~T}>\mathrm{C}$, c. $571 \mathrm{~T}>\mathrm{C}$, c. $597 \mathrm{C}>\mathrm{T}$, c. $1086 \mathrm{C}>\mathrm{T}, \mathrm{c} .1463 \mathrm{G}>\mathrm{C}$ and c. $\left.{ }^{* 439 T}>\mathrm{G}\right)$ in 266 Macedonians and 94 Albanians using the TaqMan allelic discrimination assay. No significant difference in the frequencies of the single nucleotide polymorphisms (SNPs) was observed between these populations. The frequency of the c.521T $>$ C SNP was the lowest $(<13.7$ and $12.2 \%$, respectively), while
\end{abstract}

\footnotetext{
${ }^{1}$ Faculty of Medicine, University "Hasan Prishtina", Blv. "Mother Theresa" NN, 10000 Prishtina, Republic of Kosovo

${ }^{2}$ Faculty of Pharmacy, Center for Biomolecular Pharmaceutical Analyses, University "Ss Cyril and Methodius" in Skopje, Blv. "Mother Theresa" 47, 1000 Skopje, Republic of Macedonia

${ }^{3}$ University Clinic of Cardiology, University "Ss Cyril and Methodius" in Skopje, Blv. "Mother Theresa" 17, 1000 Skopje, Republic of Macedonia

${ }^{4}$ Faculty of Medicine, Institute of Preclinical and Clinical Pharmacology with Toxicology, University "Ss Cyril and Methodius" in Skopje, St. "50th Division" 6, 1000 Skopje, Republic of Macedonia

${ }^{5}$ Clinic of Cardiology, University Clinical Center, University "Hasan Prishtina", Blv. "Mother Theresa" NN, 10 000, Prishtina, Republic of Kosovo
}

the frequencies of all other SNP alleles were above $40.0 \%$. Variant alleles of c. $1463 \mathrm{G}>\mathrm{C}$ and c. 1086 $\mathrm{C}>\mathrm{T}$ SNPs were not identified in either ethnic group. The haplotype analysis revealed 20 and 21 different haplotypes in the Macedonian and Albanian population, respectively. The most common haplotype in both ethnic groups, $* 1 J / * 1 K / * 1 L$, had a frequency of $39.0 \%$ and $26.6 \%$, respectively. In both populations, the variant alleles of the functionally significant c.521T $>C$ and c.388A $>$ G SNPs existed in one major haplotype $(* 15 / * 16 / * 17)$, with a frequency of 8.6 and $2.4 \%$ in the Macedonian and Albanian subjects, respectively. In conclusion, sequence variations of the $S L C O 1 B 1$ gene in the studied populations occur at high frequencies, which are similar to that of the Caucasian population. Further studies are needed to evaluate the clinical significance of these SNPs and/ or the major SLCO1B1 haplotypes they form for a large number of substrates and for susceptibility to certain diseases.

Keywords: Haplotypes; organic anion-transporting polypeptide 1B1 (OATP1B1); solute carrier organic anion-transporter family member $1 \mathrm{~B} 1$ (SLCO1B 1) gene; single nucleotide polymorphisms (SNPs); Western Balkan populations.

\section{INTRODUCTION}

Membrane influx and efflux transporters have a significant role in facilitating or preventing drug movement through biological membranes. Drug responses are largely dependent on their interplay with 
phases I and II metabolism and the physicochemical properties of a drug. They function in the selective absorption and elimination of drugs, mediate tissuespecific drug distribution and are also targets of many clinically used drugs. In addition, they play a critical role in the development of resistance to anticancer drugs, anticonvulsants and antiviral agents. When considering drug transport, two major super-families, $\mathrm{ABC}$ (ATP $b$ inding cassette) and SLC (solute carrier) transporters attract the highest scientific attention.

The SLC super family includes genes that encode facilitating transporters and ion-coupled secondary active transporters that reside in various cell membranes. Genes of the solute carrier organic anion transporter (SLCO) family encode organic aniontransporting polypeptides (OATPs), membrane influx transporters identified mostly in the intestine, liver, kidney, lung, testes, placenta and blood-brain barrier among other organs. The OATP1B1 [previously OATP2, OATP-C and liver specific transporter 1 (LST-1)], expressed in the sinusoidal membrane of the hepatocytes, is known to be involved in the hepatic uptake of a broad array of endogenous compounds (e.g., steroid conjugates, bile acids, eicosanoids and thyroid hormones) and drugs such as methotrexate, fexofenadine, repaglinide and statins [1-6]. Examples of in vitro OATP1B1 drug substrates include several HMG-CoA reductase inhibitors, angiotensin-converting enzyme inhibitors and angiotensin II receptor antagonists [6-8]. Many drugs have also been identified in vitro as OATP1B1 inhibitors and there are some in vivo interactions where OATP1B1 inhibition can be regarded as an important mechanism. Examples include cyclosporine, atorvastatin, gemfibrozil and rifampicin $[9,10]$.

The OATP1B1 protein is a 691 -amino acid glycoprotein with 12 putative membrane-spanning domains and a large fifth extracellular loop. Its encoding gene, solute carrier organic anion transporter family member 1B1 (SLCO1B1), is located on chromosome 12 (gene locus 12p12) [11]. A large number of single nucleotide polymorphisms (SNPs), both non synonymous and synonymous, have been discovered in the SLCO1B1 gene, and several of these have proven to affect a substrate-dependent transport function in vitro and in vivo $[12,13]$. While no firm evidence for association between these SNPs and development of certain diseases (e.g., gallstone development, essential hypertension) due to dysregulation of endogenous compounds transport exists, there are numerous research data pointing to their effects on drugs responses.

The SNPs $388(\mathrm{~A}>\mathrm{G})(* 1 b$, rs2306283) and $521(\mathrm{C}>\mathrm{T})(* 5$, rs4149056) are considered to be the most prevalent and most relevant variants, encoding a substitution of alanine for valine at amino acid 174 (p.Val174Ala), and amino acid change at position 130 (p.Asn130Asp), respectively. Increased transport activity of pravastatin as well as decreased plasma concentration of ezetimibe in carriers of the SLCO1B $1^{*} 1 b$ allele was observed [14,15], unlike reduced uptake of all statins except fluvastatin in hepatocytes and increased area under curve (AUC) of fexofenadine, repaglinide and irinotecan in carriers of $S L C O 1 B 1 * 5[3,4,16,17]$. The carriers of the c. $521 \mathrm{~T}>\mathrm{C}$ variant were also highlighted by a genomewide association study as a population with an increased risk for simvastatin-induced myopathy because of the increased plasma and muscle exposure to statins [18]. These findings were further confirmed by Santos et al. [19], who suggested that the SLCO1B1 genetic risk depends on the specific drug that was used. It was also shown that subjects carrying the SLCO1B1 c.388GG genotype exhibit significantly higher low-density-lipoprotein cholesterol reduction relative to c.388AA + c.388AG carriers, pointing out that the $S L C O 1 B 1 \mathrm{c} .388 \mathrm{~A}>\mathrm{G}$ polymorphism may be used as an important marker for predicting the efficacy of a lipid-lowering therapy [20].

Recent data point out that these two variants are in linkage disequilibrium (LD) and exist in variable SLCO $1 B 1$ haplotypes; AT, a haplotype known as * $1 A$ (reference haplotype), GT as $* 1 B, \mathrm{AC}$ as $* 5$ and GC as $* 15$, for c. $388 \mathrm{~A}>\mathrm{G}$ and c.521T $>\mathrm{C}$, respectively [13]. The * 15 haplotype has been consistently associated with a decreased transport activity, while controversial results have been reported for the $* 1 B$ haplotype [21]. It was also demonstrated that the SLCO1B $1 * 17$ haplotype (g. $-11187 \mathrm{G}>\mathrm{A}$, c. $388 \mathrm{G}>\mathrm{A}$ and c.521T $>$ C) was associated with increased plasma concentrations of pravastatin in humans [22], while the $* 14$ haplo type (c.388G-c.463A-c.521T) was characterized with enhanced response to fluvastatin [23].

It is becoming evident that the incidence of sequence variations in the $S L C O 1 B 1$ gene is largely dependent on the ethnic background. The c.521T $>C$ variant showed an allele frequency of approximately $10.0-15.0 \%$ in Asian populations, $10.0-20.0 \%$ 
in Caucasians and $1.0-2.0 \%$ in African-American populations. The c. $388 \mathrm{~A}>\mathrm{G}$ SNP showed an allele frequency of approximately $30.0-45.0 \%$ in Caucasians, $70.0-80.0 \%$ in African-American/Sub-Saharan African populations and $60.0-90.0 \%$ in Asian populations [12,22,24-26]. Therefore, characterization of the genetic variation in this transporting gene is an important step towards understanding the individual variation in drugs-substrates responses and developing a personalized and safer drug therapy.

To the best of our knowledge, there is no evidence about genotyping of OATP1B1 in the populations living in Western Balkans. Also, there is no evidence when considering the populations living in the whole Balkan Peninsula, with exception of one report evaluating association between three SLCO1B1 SNPs and statin response in the Greek population [27]. In this respect, there has not been any report on the genotype of $S L C O 1 B 1$ allelic variants in Macedonian and Albanian populations who are considered Caucasians. The origin of the Macedonians and Albanians is a continuing matter of discussion among historians; they also showed unequivocal signs of a common genetic history. In addition, Western Balkan countries have always been a historical crossroads between Asia, Africa and Europe. Considering all the above, the overall aim was to analyze the diversity of the SLCO1B1 gene in selected ethnically diverse populations living in the Western Balkans [Republic of Macedonia (RoM) and Republic of Kosovo (RoK)]. In this article, the results from the allele and genotypic frequencies of the several known SNPs in the $S L C O 1 B 1$ gene and the haplotypes they form are presented. The results from this study could serve as a baseline clinical data for dosing of all drugs substrates of OATAP1B1 and avoiding the adverse drug reactions.

\section{MATERIALS AND METHODS}

Subjects and Study Protocol. For the aim of this study, a total of 233 Caucasian patients (age 18-72 years, average body mass index (BMI) $26.20 \mathrm{~kg} / \mathrm{m}^{2}$, 109 women and 124 men) with hypercholesterolemia type IIa or IIb, were selected randomly from the outpatients evaluated for coronary heart disease at the University Clinic of Cardiology in Skopje (RoM) and the University Clinical Center in Prishtina, Clinic for Internal Diseases (RoK). Of these, 156 (66.95\%) were
Macedonians, 64 (27.47\%) Albanians, four (1.72\%) Turks and nine (3.86\%) Gypsies. Due to the low number of patients, the data for the groups of Turks and Gypsies are not presented in this paper. Therefore, the evaluated group of patients (220 individuals, 105 female and 115 male patients) consisted of $70.91 \%$ Macedonians ( $n=156,73$ women and 83 men) and $29.09 \%$ Albanians ( $n=64,32$ women and 32 men).

Initially, the study protocol was approved by the Ethics Committee of the Faculty of Pharmacy and Committee for Clinical Studies of the Faculty of Medicine, University "Ss. Cyril and Methodius" (UKIM), Skopje, RoM, and the Ethics Committee and Committee for Clinical Studies of the Faculty of Medicine, University in Prishtina, RoK. All participants received oral and written information and gave a written informed consent before entering the study. Exclusion criteria (note: not relevant for the results present in this study, but important for the overall aim of the research) included cancer in remission for period shorter than 5 years, Cushing syndrome, hyperthyroidism, positive hepatitis B surface antigen, hepatitis $\mathrm{C}$ virus antibody, fibromyalgia, myopathy, rhabdomyolysis, malabsorption syndrome, renal failure, liver disease, McArdle disease, women who are pregnant, nursing or have planned a pregnancy, drugs interacting at the level of SLCO1B1. Data for BMI, cigarette smoking, blood pressure, alcohol consumption, physical activity and pharmacotherapy were also collected and recorded. To evaluate the frequency of genetic variations in genes encoding $S L C O 1 B 1$, one blood sample was obtained from each participant for DNA extraction on the first day of the hospital visit.

In this study, 140 DNA samples obtained from the DNA bank of the Center for Biomolecular Analysis at the UKIM-Faculty of Pharmacy, Skopje, RoM, were also analyzed for the diversity of the SLCO1B1 gene. These samples were obtained from healthy individuals (of Caucasian ethnicity, $78.57 \%$ Macedonians, 21.43\% Albanians, 79 males, average age $48.0 \pm 12.9$, BMI $26.16 \mathrm{~kg} / \mathrm{m}^{2}$ ) selected by medical history, physical examination and routine laboratory tests before entering the study. Considering that there was no significant difference $(p>0.05)$ in the allelic frequencies of SLCO1B1 variants and genotype distributions between healthy subjects and patient groups, statistical analysis was also performed on the total population consisted of 360 subjects, of which $73.89 \%$ were Macedonians $(n=266,129$ 
women and $137 \mathrm{men})$ and $26.11 \%$ Albanians $(n=94$, 42 women and 52 men).

Genomic DNA Extraction and Genotyping Procedures. Three $\mathrm{mL}$ venous blood samples drawn with EDTA as anticoagulant were collected and stored at $4{ }^{\circ} \mathrm{C}$ prior to DNA isolation. DNA isolation was performed at the Center for Biomolecular Pharmaceutical Analyses, UKIM-Faculty of Pharmacy, Skopje, RoM, using the Qiamp DNA Blood kit (Qiagen GmbH, Hilden, Germany) according to the manufacturer's protocol. The samples were kept at $-20{ }^{\circ} \mathrm{C}$ until further analysis. The SLCO1B1 SNPs to be genotyped were selected on the basis of literature data $[6,13,20,28,29]$ and a previous study in which 151 subjects were included [30]. The following variants in the $S L C O 1 B 1$ gene were analyzed: c. $388 \mathrm{~A}>\mathrm{G}$ (Asn130Asp, rs2306283), c.521T $>\mathrm{C}$ (Val174Ala, rs4149056), c.571T $>$ C (Leu191Leu, rs414 9057), c.597C $>$ T (Phe199Phe, rs2291075), c.1086C $>$ T (Tyr362Tyr, rs57040246), c.1463G $>$ C (Gly488Ala, rs5950 2379), c.*439T $>\mathrm{G}$ (rs4149087, the position is given with the first nucleotide 3 ' of the stop codon (TAA) set to *1) using TaqMan allelic discrimination assay (Applied Bio-systems, Foster City, CA, USA).

Polymerase chain reaction was performed on the quantitative real-time PCR (q-PCR) system Mx3005P (Strata gene, La Jolla, CA, USA) using TaqMan genotyping protocols (TaqMan ${ }^{\circledR}$ Drug Metabolizing assay; Applied Bio-systems) in total volume of 12.5 $\mu \mathrm{L}$ under following conditions: one cycle of $2 \mathrm{~min}$. at $50{ }^{\circ} \mathrm{C}$, one cycle of $10 \mathrm{~min}$. at $95^{\circ} \mathrm{C}$, and 50 cycles of 15 seconds at $92^{\circ} \mathrm{C}$ and $1 \mathrm{~min}$. at $60^{\circ} \mathrm{C}$.

Population Genetics and Statistical Analysis. The study sample alleles and genotype frequencies were estimated with a gene counting method. The agreement with Hardy-Weinberg equilibrium (HWE) of the observed genotypic distribution for the SLCOIB1 gene was tested with the $\chi^{2}$ test. The statistical analysis was carried out using the Statistical Package for the Social Sciences (SPSS Inc., Chicago, IL, USA) software (v. 19.0).

Genetic diversity was quantified between the members of the same ethnic population, between the ethnic populations, and between different ethnic populations and the global population. Population comparisons were also performed with the $\chi^{2}$ test of population differentiation. Odds ratios (ORs) were calculated with $95 \%$ confidence interval $(95 \% \mathrm{CI})$. For multiple comparisons, Bonferroni's post hoc test was used. Statistically significant differences were those where the $p$ value was less 0.05 . Linkage disequilibrium for each pair of SNPs within each population was quantified (correlation $r^{2}$ and coef-

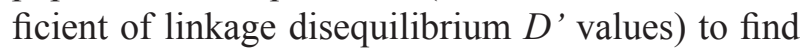
the haplotypes in the study groups. The statistical analyses were carried out using the SHEsis software platform for the analysis of LD, haplotype construction and genetic association at polymorphism loci (http://analysis2.bio-X.cn/myAnalysis.php) [31]. The haplotypes were presented with their previously assigned names, as cited in the study of Pasanen et al. [13] in which allelic frequencies at 11 variant sites were determined (g.11187G $>$ A, g.11110T $>\mathrm{G}$, g. $10499 \mathrm{~A}>\mathrm{C}$, c. $388 \mathrm{~A}>\mathrm{G}$, c. $411 \mathrm{G}>\mathrm{A}$, c. $463 \mathrm{C}>\mathrm{A}$, c. $521 \mathrm{~T}>\mathrm{C}$, c. $571 \mathrm{~T}>\mathrm{C}, \mathrm{c} .597 \mathrm{C}>\mathrm{T}, \mathrm{c} .1929 \mathrm{~A}>\mathrm{C}$ and c. $\left.{ }^{*} 439 \mathrm{~T}>\mathrm{G}\right)$. Considering that five of these SNPs and two other SNPs have been analyzed in the present investigation, one haplotype has several names and there are haplotypes that we designated as new.

\section{RESULTS}

Genotypes and Allele Frequencies. Genetic variation of SLCO1B1 was studied in 360 subjects in total, both patients with hyperlipidemia type IIa or IIb and healthy subjects, of which 266 were of Macedonian and 94 of Albanian ethnicity. Observed genotypes and allelic frequencies of $S L C O 1 B 1$ gene polymorphisms did not differ significantly $(p>0.05)$ when comparing the data obtained from patients and healthy subjects (Table 1). In addition, the observed frequency distributions did not show significant deviations from HWE $(p>0.05)$ in both populations of the two ethnic groups, the population of both patients and healthy subjects, confirming the random selection of the individuals, i.e., representativeness of the population samples being studied. Taking all this into consideration, genotype and allele frequencies for the total population of Macedonians and Albanians were estimated and the data are presented in Table 2.

Data for distribution of genotypes and allele frequencies of SLCO1B1 gene polymorphisms between females and males, including both patients and healthy subjects within each ethnic group, are presented in Table 3. No significant differences for all SLCO1B1 gene polymorphisms were observed between female and male subjects, both within each and between the two ethnic groups. 
BALKAN JOURNAL OF MEDICAL GENETICS

Daka Grapci A, Dimovski AJ, Kapedanovska A, Vavlukis M, Eftimov A, Matevska Geshkovska N, Labachevski N, Jakjovski K, Gorani D, Kedev S, Mladenovska K

Table 1. Allelic and genotypic frequencies of SLCOIB1 in patients with hyperlipidemia type IIa or IIb and healthy subjects.

\begin{tabular}{|c|c|c|c|c|}
\hline \multirow{2}{*}{$\begin{array}{l}\text { Ethnic Group } \\
\text { Number of Subjects }\end{array}$} & \multicolumn{2}{|c|}{ Macedonian $^{\mathrm{a}}$} & \multicolumn{2}{|c|}{ Albanian $^{\text {b }}$} \\
\hline & Patients $(n=156)(\%)$ & $\begin{array}{l}\text { Healthy Subjects } \\
(n=110)(\%)\end{array}$ & Patients $(n=64)(\%)$ & $\begin{array}{c}\text { Healthy Subjects } \\
(n=30)(\%)\end{array}$ \\
\hline \multicolumn{5}{|c|}{ Location/Position/dbSNP ID } \\
\hline \multicolumn{5}{|c|}{ Exon 4/c.388A>G/rs2306283 } \\
\hline $\mathrm{AA}$ & $54(34.6)$ & $34(30.9)$ & $20(31.2)$ & $9(30.0)$ \\
\hline $\mathrm{AG}$ & $80(51.3)$ & $58(52.7)$ & $35(54.7)$ & $16(53.3)$ \\
\hline GG & $22(14.1)$ & $18(16.3)$ & $9(14.1)$ & $5(16.7)$ \\
\hline$p$ Value $^{\mathrm{d}}$ & \multicolumn{2}{|c|}{0.77369} & \multicolumn{2}{|c|}{0.94636} \\
\hline G allele & $124(40.0)$ & $94(42.7)$ & $53(41.4)$ & $26(43.3)$ \\
\hline A allele & $188(60.0)$ & $126(57.3)$ & $75(58.6)$ & $34(56.7)$ \\
\hline$p$ Value $^{\mathrm{e}}$ & \multicolumn{2}{|c|}{0.49074} & \multicolumn{2}{|c|}{0.80295} \\
\hline \multicolumn{5}{|c|}{ Exon 5/c.521T >C/rs4149056 } \\
\hline $\mathrm{CC}$ & $4(2.6)$ & $5(4.5)$ & - & $1(3.0)$ \\
\hline CT & $36(23.1)$ & $19(17.3)$ & $14(21.9)$ & $7(23.3)$ \\
\hline TT & $116(74.3)$ & $86(78.2)$ & $50(78.1)$ & $22(73.3)$ \\
\hline$p$ Value $^{\mathrm{d}}$ & \multicolumn{2}{|c|}{0.38218} & \multicolumn{2}{|c|}{0.33055} \\
\hline $\mathrm{C}$ allele & $44(14.1)$ & $29(13.2)$ & $14(11.0)$ & $9(15.0)$ \\
\hline T allele & $268(85.9)$ & $191(86.8)$ & $114(89.0)$ & $51(85.0)$ \\
\hline$p$ Value $^{\mathrm{e}}$ & \multicolumn{2}{|c|}{0.76116} & \multicolumn{2}{|c|}{0.42813} \\
\hline \multicolumn{5}{|c|}{ Exon 5/c.571T >C/rs4149057 } \\
\hline $\mathrm{CC}$ & $66(42.3)$ & $45(41.0)$ & $22(34.4)$ & $12(40.0)$ \\
\hline CT & $71(45.5)$ & $53(48.2)$ & $22(53.1)$ & $15(50.0)$ \\
\hline TT & $19(12.2)$ & $12(11.0)$ & $8(12.5)$ & $3(10.0)$ \\
\hline$p$ Value $^{\mathrm{d}}$ & \multicolumn{2}{|c|}{0.89666} & \multicolumn{2}{|c|}{0.84958} \\
\hline $\mathrm{C}$ allele & $203(65.0)$ & $143(65.0)$ & $78(61.0)$ & $39(65.0)$ \\
\hline T allele & $109(35.0)$ & $77(35.0)$ & $50(39.0)$ & $21(35.0)$ \\
\hline$p$ Value $^{\mathrm{e}}$ & \multicolumn{2}{|c|}{0.98782} & \multicolumn{2}{|c|}{0.59224} \\
\hline \multicolumn{5}{|c|}{ Exon 5/c.597C > T/rs229107 } \\
\hline $\mathrm{CC}$ & $60(38.5)$ & $40(36.4)$ & $16(25.0)$ & $11(36.7)$ \\
\hline CT & $67(42.9)$ & $50(45.5)$ & $35(54.7)$ & $14(46.6)$ \\
\hline TT & $29(18.6)$ & $20(18.2)$ & $13(20.3)$ & $5(16.7)$ \\
\hline$p$ Value $^{\mathrm{d}}$ & \multicolumn{2}{|c|}{0.91693} & \multicolumn{2}{|c|}{0.50617} \\
\hline T allele & $125(40.1)$ & $90(40.9)$ & $61(47.6)$ & $24(40.0)$ \\
\hline $\mathrm{C}$ allele & $187(59.9)$ & $130(59.1)$ & $67(52.3)$ & $36(40.0)$ \\
\hline$p$ Value $^{\mathrm{e}}$ & \multicolumn{2}{|c|}{0.84493} & \multicolumn{2}{|c|}{0.32550} \\
\hline \multicolumn{5}{|c|}{ Exon 8/c.1086C > T/rs57040246 } \\
\hline $\mathrm{CC}$ & $156(100.0)$ & $110(100.0)$ & $64(100.0)$ & $30(100.0)$ \\
\hline $\mathrm{CT}$ & $0(0.0)$ & $0(0.0)$ & $0(0.0)$ & $0(0.0)$ \\
\hline TT & $0(0.0)$ & $0(0.0)$ & $0(0.0)$ & $0(0.0)$ \\
\hline$p$ Value $^{\mathrm{d}}$ & \multicolumn{2}{|c|}{$>0.05$} & & \\
\hline T allele & $0(0.0)$ & $0(0.0)$ & $0(0.0)$ & $0(0.0)$ \\
\hline$p$ Value & & & & \\
\hline
\end{tabular}




\section{BJMG}

Table 1. Continued

\begin{tabular}{|c|c|c|c|c|}
\hline \multicolumn{5}{|c|}{ Exon 10/c.1463G >C/rs59502379 } \\
\hline $\mathrm{CC}$ & $0(0.0)$ & $0(0.0)$ & $0(0.0)$ & $0(0.0)$ \\
\hline $\mathrm{CG}$ & $0(0.0)$ & $0(0.0)$ & $0(0.0)$ & $0(0.0)$ \\
\hline GG & $156(100.0)$ & $58(100.0)$ & $64(100.0)$ & $30(100.0)$ \\
\hline$p$ Value $^{\mathrm{d}}$ & \multicolumn{2}{|c|}{$>0.05$} & \multicolumn{2}{|c|}{$>0.05$} \\
\hline $\mathrm{C}$ allele & $0(0.0)$ & $0(0.0)$ & $0(0.0)$ & $0(0.0)$ \\
\hline$p$ Value $^{\mathrm{e}}$ & \multicolumn{2}{|c|}{$>0.05$} & \multicolumn{2}{|c|}{$>0.05$} \\
\hline \multicolumn{5}{|c|}{ 3'UTR/c.*439T>G/rs4149087 } \\
\hline GG & $32(20.5)$ & $28(25.45)$ & $15(23.4)$ & $9(30.0)$ \\
\hline GT & $81(51.9)$ & $50(45.45)$ & $31(48.4)$ & $16(53.3)$ \\
\hline $\mathrm{TT}$ & $43(27.6)$ & $32(29.1)$ & $18(28.1)$ & $5(16.7)$ \\
\hline$p$ Value $^{\mathrm{d}}$ & \multicolumn{2}{|c|}{0.52208} & \multicolumn{2}{|c|}{0.46337} \\
\hline G allele & $145(46.5)$ & $106(48.2)$ & $61(47.7)$ & $34(56.7)$ \\
\hline T allele & $167(53.5)$ & $114(51.8)$ & $67(52.3)$ & $26(43.3)$ \\
\hline$p$ Value $^{\mathrm{e}}$ & \multicolumn{2}{|c|}{0.69853} & \multicolumn{2}{|c|}{0.24882} \\
\hline
\end{tabular}

dbSNP: database of single nucleotide polymorphism; 3'UTR: 3' untranslated region; NCBI: National Center for Biotechnology Information.

a Macedonians populating the RoM.

${ }^{\mathrm{b}}$ Albanians populating the RoM and RoK.

${ }^{c}$ The positions of SNPs are given in relation to the NCBI reference sequences NM_006446.2 (cDNA; c.) with the fist nucleotide of the ATG first codon set to 1 and the nucleotide 5 ' of ATG set to -1 . The position of c.*439 is given with the first nucleotide 3 ' of the stop codon (TAA) set to *1.

${ }^{\mathrm{d}}$ The $p$ value for the differences of genotype distributions between the patients and healthy subjects within the ethnic group.

${ }^{\mathrm{e}}$ The $p$ value for the differences of allelic frequencies between the patients and healthy subjects within the ethnic group.

Table 2. Genetic variation of the SLCO1B1 gene in Macedonian and Albanian subjects.

\begin{tabular}{|c|c|c|c|c|c|c|}
\hline \multirow{2}{*}{$\begin{array}{l}\text { Ethnic Group } \\
\text { Number of Subjects }\end{array}$} & \multicolumn{3}{|c|}{ Macedonian $^{\mathrm{a}}$} & \multicolumn{3}{|c|}{ Albanian $^{\mathrm{b}}$} \\
\hline & $\begin{array}{c}\text { Observed } \\
\text { Frequency } \\
n=266(\%)\end{array}$ & $\begin{array}{c}\text { Expected } \\
\text { Frequency by } \\
\text { HWE (\%) }\end{array}$ & $p$ Value $^{\mathrm{c}}$ & $\begin{array}{c}\text { Observed } \\
\text { Frequency } \\
n=94(\%)\end{array}$ & $\begin{array}{c}\text { Expected } \\
\text { Frequency by } \\
\text { HWE (\%) }\end{array}$ & $p$ Value $^{\mathrm{c}}$ \\
\hline \multicolumn{7}{|l|}{ Position $/ \mathrm{dbSNP}$ ID } \\
\hline \multicolumn{7}{|l|}{ c. $388 \mathrm{~A}>\mathrm{G} / \mathrm{rs} 2306283$} \\
\hline AA & $88(33.1)$ & 34.8 & & $29(30.8)$ & 33.6 & \\
\hline $\mathrm{AG}$ & $138(51.9)$ & 48.4 & 0.99737 & $51(54.2)$ & 48.7 & 0.99358 \\
\hline GG & $40(15.0)$ & 16.8 & & $14(14.9)$ & 17.7 & \\
\hline \multicolumn{3}{|l|}{$p$ Value $^{\mathrm{e}}$} & 0.91299 & & & \\
\hline $\mathrm{G}$ allele & $218(40.9)$ & & & $79(42.0)$ & & \\
\hline A allele & $314(59.0)$ & & & $109(58.0)$ & & \\
\hline \multicolumn{3}{|l|}{$p$ Value $^{\mathrm{f}}$} & 0.80266 & & & \\
\hline \multicolumn{7}{|l|}{ c. $521 \mathrm{~T}>\mathrm{C} / \mathrm{rs} 4149056$} \\
\hline $\mathrm{CC}$ & $9(3.4)$ & 1.9 & & $1(1.1)$ & 1.5 & \\
\hline $\mathrm{CT}$ & $55(20.7)$ & 23.7 & 0.99200 & $21(22.3)$ & 21.5 & 0.99919 \\
\hline $\mathrm{TT}$ & $202(75.9)$ & 74.4 & & $72(76.6)$ & 77.0 & \\
\hline \multicolumn{3}{|l|}{$p$ Value $^{\mathrm{e}}$} & 0.48666 & & & \\
\hline $\mathrm{C}$ allele & $73(13.7)$ & & & $23(12.2)$ & & \\
\hline $\mathrm{T}$ allele & $459(86.3)$ & & & $165(87.8)$ & & \\
\hline \multicolumn{3}{|l|}{$p$ Value $^{\mathrm{f}}$} & 0.60597 & & & \\
\hline
\end{tabular}


BALKAN JOURNAL OF MEDICAL GENETICS

Daka Grapci A, Dimovski AJ, Kapedanovska A, Vavlukis M, Eftimov A, Matevska Geshkovska N, Labachevski N, Jakjovski K, Gorani D, Kedev S, Mladenovska K

Table 2. Continued

\begin{tabular}{|c|c|c|c|c|c|c|}
\hline \multicolumn{7}{|c|}{ c. $571 \mathrm{~T}>\mathrm{C} / \mathrm{rs} 4149057$} \\
\hline $\mathrm{CC}$ & $111(41.7)$ & 42.3 & & $34(36.2)$ & 38.7 & \\
\hline $\mathrm{CT}$ & $124(46.6)$ & 45.5 & 0.99969 & $49(52.1)$ & 47.0 & 0.99408 \\
\hline TT & $31(11.6)$ & 12.2 & & $11(11.7)$ & 14.3 & \\
\hline \multicolumn{3}{|l|}{$p$ Value $^{\mathrm{e}}$} & 0.61507 & & & \\
\hline $\mathrm{C}$ allele & $346(65.0)$ & & & $117(62.2)$ & & \\
\hline T allele & $186(35.0)$ & & & $71(37.8)$ & & \\
\hline \multicolumn{3}{|l|}{$p$ Value $^{\mathrm{f}}$} & 0.49039 & & & \\
\hline \multicolumn{7}{|c|}{ c. $597 \mathrm{C}>\mathrm{T} / \mathrm{rs} 229107$} \\
\hline $\mathrm{CC}$ & $100(37.6)$ & 35.5 & & $27(28.7)$ & 30.0 & \\
\hline $\mathrm{CT}$ & $117(44.0)$ & 48.2 & 0.99625 & $49(52.1)$ & 49.5 & 0.99864 \\
\hline TT & $49(18.4)$ & 16.3 & & $18(19.1)$ & 20.4 & \\
\hline \multicolumn{3}{|l|}{$p$ Value $^{\mathrm{e}}$} & 0.27697 & & & \\
\hline T allele & $215(40.4)$ & & & $85(45.2)$ & & \\
\hline $\mathrm{C}$ allele & $317(59.6)$ & & & $103(54.8)$ & & \\
\hline \multicolumn{3}{|l|}{$p$ Value $^{\mathrm{f}}$} & 0.25125 & & & \\
\hline \multicolumn{7}{|c|}{ c. $1086 \mathrm{C}>\mathrm{T} / \mathrm{rs} 57040246$} \\
\hline $\mathrm{CC}$ & $266(100.0)$ & 100.0 & & $94(100.0)$ & 100.0 & \\
\hline $\mathrm{CT}$ & $0(0.0)$ & 0.0 & $>0.05$ & $0(0.0)$ & 0.0 & $>0.05$ \\
\hline TT & $0(0.0)$ & 0.0 & & $0(0.0)$ & 0.0 & \\
\hline \multicolumn{3}{|l|}{$p$ Value $^{\mathrm{e}}$} & $>0.05$ & & & \\
\hline T allele & $0(0.0)$ & 0.0 & & $0(0.0)$ & & \\
\hline \multicolumn{3}{|l|}{$p$ Value $^{\mathrm{f}}$} & $>0.05$ & & & \\
\hline \multicolumn{7}{|c|}{ c. $1463 \mathrm{G}>\mathrm{C} / \mathrm{rs} 59502379$} \\
\hline $\mathrm{CC}$ & $0(0.0)$ & $0(0.0)$ & & $0(0.0)$ & $0(0.0)$ & \\
\hline $\mathrm{CG}$ & $0(0.0)$ & $0(0.0)$ & $>0.05$ & $0(0.0)$ & $0(0.0)$ & $>0.05$ \\
\hline GG & $266(100.0)$ & 100.0 & & $94(100.0)$ & 100.0 & \\
\hline \multicolumn{3}{|l|}{$p$ Value $^{\mathrm{e}}$} & $>0.05$ & & & \\
\hline $\mathrm{C}$ allele & $0(0.0)$ & & & $0(0.0)$ & & \\
\hline \multicolumn{3}{|l|}{$p$ Value $^{\mathrm{f}}$} & $>0.05$ & & & \\
\hline \multicolumn{7}{|c|}{ c. $* 439 \mathrm{~T}>\mathrm{G} / \mathrm{rs} 4149087$} \\
\hline GG & $60(22.5)$ & 25.5 & & $24(25.5)$ & 22.2 & \\
\hline GT & $131(49.2)$ & 50.0 & 0.99993 & $47(50.0)$ & 49.8 & 1.0000 \\
\hline TT & $75(28.2)$ & 24.5 & & $23(24.5)$ & 27.9 & \\
\hline \multicolumn{3}{|l|}{$p$ Value $^{\mathrm{e}}$} & 0.73126 & & & \\
\hline G allele & $251(47.2)$ & & & $95(50.5)$ & & \\
\hline $\mathrm{T}$ allele & $281(52.8)$ & & & 93 (49.5) & & \\
\hline \multicolumn{3}{|l|}{$p$ Value $^{\mathrm{f}}$} & 0.42917 & & & \\
\hline
\end{tabular}

HWE: Hardy-Weinberg equilibrium; dbSNP: database of single nucleotide polymorphism; 3'UTR: 3' untranslated region; NCBI: National Center for Biotechnology Information.

${ }^{a}$ Macedonians populating the RoM.

${ }^{\mathrm{b}}$ Albanians populating the RoM and RoK.

${ }^{c}$ The $p$ value for the differences between observed and expected frequencies of genotype distributions within the ethnic group.

${ }^{d}$ The positions of SNPs are given in relation to the NCBI reference sequences NM 006446.2 (cDNA; c.) with the fist nucleotide of the ATG first

codon set to 1 and the nucleotide 5 ' of ATG set to -1 . The position of c.*439 is given with the first nucleotide 3 ' of the stop codon (TAA) set to *1.

e The $p$ value of differences in genotype distributions between the ethnic groups.

${ }^{\mathrm{f}}$ The $p$ value of allele frequencies between the ethnic groups. 


\section{BJMG}

SNPs OF THE SLCO1B1 GENE

Table 3. Distribution of genotype and allele frequencies of the SLCO1B1 gene polymorphisms in female and male groups separately, within each ethnic group.

\begin{tabular}{|c|c|c|c|c|}
\hline \multirow{2}{*}{$\begin{array}{l}\text { Ethnic Group } \\
\text { Number of Subjects }\end{array}$} & \multicolumn{2}{|c|}{ Macedonian } & \multicolumn{2}{|c|}{ Albanian } \\
\hline & Females $(n=129)(\%)$ & Males $(n=137)(\%)$ & Females $(n=42)(\%)$ & Males $(n=52)(\%)$ \\
\hline \multicolumn{5}{|l|}{ Position/dbSNP ID } \\
\hline \multicolumn{5}{|l|}{ c. $388 \mathrm{~A}>\mathrm{G} / \mathrm{rs} 2306283$} \\
\hline $\mathrm{AA}$ & $42(32.6)$ & $46(33.6)$ & $14(33.3)$ & $15(28.8)$ \\
\hline $\mathrm{AG}$ & $65(50.4)$ & $73(53.3)$ & $24(57.1)$ & $27(51.9)$ \\
\hline GG & $22(17.0)$ & $18(13.1)$ & $4(9.5)$ & $10(19.2)$ \\
\hline$p$ Value $^{\mathrm{a}}$ & \multicolumn{2}{|c|}{0.66841} & \multicolumn{2}{|c|}{0.41932} \\
\hline G allele & $109(42.2)$ & $109(39.8)$ & $32(38.1)$ & $47(45.2)$ \\
\hline A allele & $149(57.8)$ & $165(60.2)$ & $92(61.9)$ & $57(54.8)$ \\
\hline$p$ Value $^{\mathrm{b}}$ & \multicolumn{2}{|c|}{0.56309} & \multicolumn{2}{|c|}{0.32702} \\
\hline \multicolumn{5}{|l|}{ c. $521 \mathrm{~T}>\mathrm{C} / \mathrm{rs} 4149056$} \\
\hline $\mathrm{CC}$ & $6(4.6)$ & $3(2.2)$ & - & $1(2.0)$ \\
\hline $\mathrm{CT}$ & $25(19.4)$ & $30(21.9)$ & $9(21.4)$ & $12(23.1)$ \\
\hline TT & $98(76.0)$ & $104(75.9)$ & $33(78.6)$ & $39(75.0)$ \\
\hline$p$ Value $^{\mathrm{a}}$ & \multicolumn{2}{|c|}{0.49822} & \multicolumn{2}{|c|}{0.64576} \\
\hline $\mathrm{C}$ allele & $37(14.3)$ & $36(13.1)$ & $9(10.7)$ & $14(13.5)$ \\
\hline T allele & $221(85.7)$ & $238(86.9)$ & $75(89.3)$ & $90(86.5)$ \\
\hline$p$ Value $^{\mathrm{b}}$ & \multicolumn{2}{|c|}{0.68707} & \multicolumn{2}{|c|}{0.56765} \\
\hline \multicolumn{5}{|l|}{ c. $571 \mathrm{~T}>\mathrm{C} / \mathrm{rs} 4149057$} \\
\hline $\mathrm{CC}$ & $53(41.2)$ & $58(42.3)$ & $14(33.3)$ & $20(38.5)$ \\
\hline $\mathrm{CT}$ & $61(47.3)$ & $63(46.0)$ & $23(54.8)$ & $26(50.0)$ \\
\hline TT & $15(11.6)$ & $16(11.7)$ & $5(11.9)$ & $6(11.5)$ \\
\hline$p$ Value $^{\mathrm{a}}$ & \multicolumn{2}{|c|}{0.97571} & \multicolumn{2}{|c|}{0.82901} \\
\hline $\mathrm{C}$ allele & $167(64.7)$ & $179(65.3)$ & $51(60.7)$ & $66(63.5)$ \\
\hline T allele & $91(35.3)$ & $95(34.7)$ & $33(39.3)$ & $38(36.5)$ \\
\hline$p$ Value $^{\mathrm{b}}$ & \multicolumn{2}{|c|}{0.88472} & \multicolumn{2}{|c|}{0.69928} \\
\hline \multicolumn{5}{|l|}{ c.597C > T/rs229107 } \\
\hline $\mathrm{CC}$ & $49(38.0)$ & $51(37.2)$ & $12(28.6)$ & $15(28.8)$ \\
\hline $\mathrm{CT}$ & $57(44.2)$ & $60(43.8)$ & $22(52.4)$ & $27(51.9)$ \\
\hline TT & $23(17.8)$ & $26(19.0)$ & $8(19.0)$ & $10(19.2)$ \\
\hline$p$ Value $^{\mathrm{a}}$ & \multicolumn{2}{|c|}{0.97042} & \multicolumn{2}{|c|}{0.99902} \\
\hline $\mathrm{C}$ allele & $103(39.9)$ & $112(40.9)$ & $38(45.2)$ & $47(45.2)$ \\
\hline T allele & $155(60.1)$ & $162(59.1)$ & $46(54.8)$ & $57(54.8)$ \\
\hline$p$ Value $^{\mathrm{b}}$ & \multicolumn{2}{|c|}{0.82278} & \multicolumn{2}{|c|}{0.99500} \\
\hline c. $1086 \mathrm{C}>\mathrm{T} / \mathrm{rs} 5704024$ & & & & \\
\hline $\mathrm{CC}$ & $129(100.0)$ & $137(100.0)$ & $42(100.0)$ & $52(100.0)$ \\
\hline $\mathrm{CT}$ & $0(0.0)$ & $0(0.0)$ & $0(0.0)$ & $0(0.0)$ \\
\hline TT & $0(0.0)$ & $0(0.0)$ & $0(0.0)$ & $0(0.0)$ \\
\hline T allele & $0(0.0)$ & $0(0.0)$ & $0(0.0)$ & $0(0.0)$ \\
\hline c. $1463 \mathrm{G}>\mathrm{C} / \mathrm{rs} 595023$ & & & & \\
\hline $\mathrm{CC}$ & $0(0.0)$ & $0(0.0)$ & $0(0.0)$ & $0(0.0)$ \\
\hline $\mathrm{CG}$ & $0(0.0)$ & $0(0.0)$ & $0(0.0)$ & $0(0.0)$ \\
\hline GG & $129(100.0)$ & $137(100.0)$ & $42(100.0)$ & $52(100.0)$ \\
\hline $\mathrm{C}$ allele & $0(0.0)$ & $0(0.0)$ & $0(0.0)$ & $0(0.0)$ \\
\hline
\end{tabular}


Table 3. Continued

\begin{tabular}{|c|c|c|c|c|}
\hline \multicolumn{5}{|c|}{ c. ${ }^{4} 439 \mathrm{~T}>\mathrm{G} / \mathrm{rs} 4149087$} \\
\hline GG & $30(23.3)$ & $30(21.9)$ & $11(26.2)$ & $13(25.0)$ \\
\hline GT & 64 (49.6) & $67(48.9)$ & $19(45.2)$ & $28(53.8)$ \\
\hline TT & $35(27.1)$ & $40(29.2)$ & $12(28.6)$ & $11(21.2)$ \\
\hline$p$ Value $^{\mathrm{a}}$ & \multicolumn{2}{|c|}{0.92239} & \multicolumn{2}{|c|}{0.64414} \\
\hline G allele & $124(48.1)$ & $127(46.4)$ & $41(48.8)$ & $54(51.9)$ \\
\hline T allele & $134(51.9)$ & $147(53.6)$ & $43(51.2)$ & $50(48.1)$ \\
\hline$p$ Value $^{\mathrm{b}}$ & \multicolumn{2}{|c|}{0.69266} & \multicolumn{2}{|c|}{0.67119} \\
\hline
\end{tabular}

a The $p$ value of differences in genotype distributions between females and males within the ethnic group.

${ }^{\mathrm{b}}$ The $p$ value of differences of allele frequencies between females and males within the ethnic group.

All SNPs, except c. $1463 \mathrm{G}>\mathrm{C}$ and c. $1086 \mathrm{C}>\mathrm{T}$, occurred at an allele frequency higher than $12.0 \%$. Variant alleles of SLCOB1 c. $1463 \mathrm{G}>\mathrm{C}$ and c. $1086 \mathrm{C}>\mathrm{T}$ polymor- phisms were not identified in either ethnic group in this study. The frequency of the c.521T $>$ C SNP was the lowest, 13.7 and $12.2 \%$ for Macedonians and Albanians, respectively, while the frequencies of all other SNPs alleles were above $40.0 \%$, with frequency of the c. $571 \mathrm{~T}>\mathrm{C}$ variant allele being highest in both populations (65.0 and 62.2\% for Macedonians and Albanians, respectively). No significant differences $(p>0.05)$ in allelic frequencies and genotype distributions of the analyzed SNPs were observed between the two ethnic groups. The SNP variant allele frequencies in the ethnic groups separately compared to data reported from various ethnic groups are presented in Table 4.

Pairwise Linkage Disequilibrium. Pairwise LD profiles for single SNPs using $r^{2}$ and $D^{\prime}$ values for Macedonians and Albanians separately, are shown in Figures 1 and 2, respectively. Generally, the correlations of SNP pairs in the Albanian population were weaker than those of the Macedonian population. The most strongly correlated $\left(r^{2} \geq 0.33\right)$ SNP pair in the Macedonian population was c.597 $\mathrm{C}>\mathrm{T} / \mathrm{c} .388 \mathrm{~A}>\mathrm{G}\left(r^{2}=0.531, D^{\prime}=0.740\right)$, followed by c. $597 \mathrm{C}>\mathrm{T} / \mathrm{c} .{ }^{*} 439 \mathrm{~T}>\mathrm{G}\left(r^{2}=0.373, D^{\prime}=0.699\right)$. Other pairs showing a significant association were c. $388 \mathrm{~A}>\mathrm{G} / \mathrm{c} . * 439 \mathrm{~T}>\mathrm{G}\left(r^{2}=0.289, D^{\prime}=0.613\right)$ and c.521T $>$ C $/$ c. $571 \mathrm{~T}>\mathrm{C}\left(r^{2}=0.233, D^{\prime}=0.919\right)$. The correlation of the most common SNP pair, c.388A $>\mathrm{G} /$ c. $521 \mathrm{~T}>\mathrm{C}$, in the Macedonians was relatively weaker compared to other SNP pairs, with $r^{2}=0.113$ and $D^{\prime}$ $=0.698$. The c.521T $>C$ showed the strongest correlation with c.571T $>\mathrm{C}$, followed by c.597 C $>\mathrm{T}\left(r^{2}=\right.$ $\left.0.178, D^{\prime}=0.872\right)$, c. $388 \mathrm{~A}>\mathrm{G}$ and c. $* 439 \mathrm{~T}>\mathrm{G}\left(r^{2}\right.$ $=0.072, D^{\prime}=0.645$ ).
In the Albanian population, the same SNP pairs, c. $597 \mathrm{C}>\mathrm{T} / \mathrm{c} .388 \mathrm{~A}>\mathrm{G}$ and c.597C $>$ T/c. $* 439 \mathrm{~T}>\mathrm{G}$, showed the strongest correlation with $r^{2}=0.221$, $D^{\prime}=0.498$ and $r^{2}=0.214, D^{\prime}=0.505$, respectively. The correlation between c. $388 \mathrm{~A}>\mathrm{G}$ and c.521T $>\mathrm{C}$ in the Albanian population was weaker $\left(r^{2}=0.009\right.$, $\left.D^{\prime}=0.219\right)$ compared to the same SNP pairs in the Macedonian population. Similar data for the LD of c.521T $>$ C with other SNPs were obtained, with the strongest correlation of this SNP with c.571T $>\mathrm{C}\left(r^{2}\right.$ $\left.=0.091, D^{\prime}=0.635\right)$, followed by c.597C $>\mathrm{T}\left(r^{2}=\right.$ $\left.0.097, D^{\prime}=0.746\right)$, c. $388 \mathrm{~A}>\mathrm{G}$ and c. $* 439 \mathrm{~T}>\mathrm{G}\left(r^{2}=\right.$ $0.008, D^{\prime}=0.238$ ).

Haplotypes. The haplotype analysis revealed 20 different haplotypes in the Macedonian population and 21 in the Albanian population (Tables 5 and 6). Nine haplotypes in each of the two populations were designated as new. Nine other haplotypes that occurred in the Macedonian and Albanian populations had the same sequence of the actually investigated SNPs as in the newly identified haplotypes presented in the study of Pasanen et al. [13].

In the Macedonian population, eight haplotypes occurred at a frequency equal to or greater than $3.0 \%$ (Table 5). The most common haplotype in this ethnic group, $* 1 J / * 1 K / * 1 L$, had a frequency of $39.0 \%$, containing variant allele $\mathrm{C}$ at position c.571 and having referent nucleotides at all other investigated positions. The variant allele $\mathrm{C}$ at position c.571 existed in eight haplotypes, with a frequency between 0.3 and $39.0 \%$. The variant allele $\mathrm{G}$ at position c. 388 and $\mathrm{T}$ at c. $597 \mathrm{C}>\mathrm{T}$ SNP existed in 11, while the variant allele $\mathrm{G}$ at c.*439T $>\mathrm{G}$ in 12 haplotypes, all occurring with frequencies between 0.3 and $11.6 \%$. The c. $521 \mathrm{~T}>\mathrm{C}$ SNP existed in six haplotypes, with a frequency between 0.3 and $8.6 \%$. The variant alleles of the functionally most distinguished SNPs, c.388A $>\mathrm{G}$ 
Table 4. Allelic frequencies of SLCO1B1 variants in Macedonians and Albanians compared to different ethnic populations.

\begin{tabular}{|c|c|c|c|c|c|c|c|c|c|c|c|}
\hline Ethnic Group & $n^{\mathrm{a}}$ & c. $388 \mathrm{~A}>\mathrm{G}$ & c.521T $>C$ & c.571T $>C$ & c.597C $>\mathrm{T}$ & c. $1086 \mathrm{C}>\mathrm{T}$ & c. $1463 \mathrm{G}>\mathrm{C}$ & c. $* 439 \mathrm{~T}>\mathrm{G}$ & $p$ Value $^{\mathrm{b}}$ & $p$ Value $^{\mathrm{c}}$ & Refs. \\
\hline $\begin{array}{l}\text { American (African) } \\
\text { (European) } \\
\text { (Native) }\end{array}$ & $\begin{array}{l}22 \\
49 \\
64\end{array}$ & $\begin{array}{l}0.75 \\
0.30 \\
0.63\end{array}$ & $\begin{array}{c}0.023 \\
0.14 \\
0.24\end{array}$ & $\begin{array}{c}0.045 \\
0.53 \\
0.33\end{array}$ & $\begin{array}{c}- \\
- \\
0.28\end{array}$ & $\begin{array}{c}- \\
- \\
0.01\end{array}$ & $\begin{array}{c}0.09 \\
0.0 \\
0.005\end{array}$ & $\begin{array}{c}- \\
- \\
0.041\end{array}$ & $\begin{array}{c}<0.00001 \\
0.812508 \\
0.000507\end{array}$ & $\begin{array}{c}<0.00001 \\
0.675274 \\
0.003258\end{array}$ & $\begin{array}{l}12 \\
12 \\
11\end{array}$ \\
\hline $\begin{array}{l}\text { European } \\
\text { (Caucasian) }\end{array}$ & $\begin{array}{l}151 \\
236\end{array}$ & $\begin{array}{l}0.41 \\
0.41\end{array}$ & $\begin{array}{l}0.18 \\
0.17\end{array}$ & $\begin{array}{c}0.61 \\
-\end{array}$ & $\begin{array}{c}0.42 \\
-\end{array}$ & $\begin{array}{c}0.0 \\
-\end{array}$ & $\begin{array}{c}0.0 \\
-\end{array}$ & $\begin{array}{c}0.30 \\
-\end{array}$ & $\begin{array}{l}0.118756 \\
0.466510\end{array}$ & $\begin{array}{l}0.149653 \\
0.328042\end{array}$ & $\begin{array}{l}11 \\
38\end{array}$ \\
\hline Sub-Saharan African & 105 & 0.79 & 0.019 & 0.13 & 0.50 & 0.07 & 0.03 & 0.76 & $<0.00001$ & $<0.00001$ & 11 \\
\hline Oceanian & 28 & 0.66 & 0.0 & 0.48 & 0.52 & 0.036 & 0.0 & 0.30 & 0.017744 & 0.055677 & 11 \\
\hline Algerian & 29 & 0.64 & 0.17 & 0.21 & 0.59 & 0.017 & 0.0 & 0.72 & 0.004001 & 0.017564 & 11 \\
\hline Ugandan & 115 & 0.78 & 0.039 & 0.061 & 0.0 & - & 0.02 & - & $<0.00001$ & $<0.00001$ & 11 \\
\hline $\begin{array}{l}\text { Indian (Asian) } \\
\text { North Indian }\end{array}$ & $\begin{array}{c}35 \\
100\end{array}$ & $\begin{array}{l}0.60 \\
0.57\end{array}$ & $\begin{array}{l}0.071 \\
0.065\end{array}$ & $\begin{array}{c}- \\
0.44\end{array}$ & $\begin{array}{c}- \\
0.22\end{array}$ & $\begin{array}{l}0.0 \\
0.0\end{array}$ & - & - & $\begin{array}{l}0.078203 \\
0.000010\end{array}$ & $\begin{array}{l}0.167511 \\
0.009301\end{array}$ & $\begin{array}{l}24 \\
25\end{array}$ \\
\hline $\begin{array}{l}\text { Brazilian } \\
\text { (African) } \\
\text { (Mulatto) } \\
\text { (Caucasian) } \\
\text { (Amerindian) Brazilian }\end{array}$ & $\begin{array}{r}97 \\
332 \\
603 \\
182 \\
143\end{array}$ & $\begin{array}{l}- \\
- \\
- \\
- \\
0.26\end{array}$ & $\begin{array}{c}0.057 \\
0.15 \\
0.15 \\
0.28 \\
0.14\end{array}$ & $\begin{array}{l}- \\
- \\
- \\
- \\
-\end{array}$ & $\begin{array}{l}- \\
- \\
- \\
- \\
-\end{array}$ & $\begin{array}{l}- \\
- \\
- \\
- \\
-\end{array}$ & $\begin{array}{l}- \\
- \\
- \\
- \\
-\end{array}$ & $\begin{array}{l}- \\
- \\
- \\
- \\
-\end{array}$ & 0.164989 & 0.136491 & $\begin{array}{l}28 \\
28 \\
28 \\
28 \\
19\end{array}$ \\
\hline Chinese & $\begin{array}{c}178 \\
100 \\
35 \\
140\end{array}$ & $\begin{array}{l}0.73 \\
0.80 \\
0.67 \\
0.71\end{array}$ & $\begin{array}{c}0.11 \\
0.13 \\
0.086 \\
0.11\end{array}$ & $\begin{array}{c}0.27 \\
0.26 \\
- \\
-\end{array}$ & $\begin{array}{c}0.42 \\
0.50 \\
- \\
-\end{array}$ & $\begin{array}{l}0.0 \\
0.0 \\
- \\
-\end{array}$ & $\begin{array}{c}0.0 \\
-\end{array}$ & $\begin{array}{c}0.27 \\
-\end{array}$ & $\begin{array}{l}<0.00001 \\
<0.00001 \\
0.124778 \\
0.014801\end{array}$ & $\begin{array}{l}<0.00001 \\
<0.00001 \\
0.264367 \\
0.153581\end{array}$ & $\begin{array}{l}11 \\
25 \\
24 \\
39\end{array}$ \\
\hline Han Chinese & 111 & 0.73 & 0.14 & - & - & - & - & - & 0.079881 & 0.339359 & 29 \\
\hline Uyghur (Chinese) & 731 & 0.62 & 0.10 & - & - & - & - & - & 0.000925 & 0.119961 & 40 \\
\hline $\begin{array}{l}\text { Finnish } \\
\text { (Caucasian) }\end{array}$ & $\begin{array}{l}468 \\
193\end{array}$ & $\begin{array}{c}0.46 \\
-\end{array}$ & $\begin{array}{c}0.20 \\
-\end{array}$ & $\begin{array}{c}0.53 \\
-\end{array}$ & $\begin{array}{c}0.46 \\
-\end{array}$ & $\begin{array}{c}- \\
0.0\end{array}$ & $\begin{array}{c}- \\
0.0\end{array}$ & $\begin{array}{c}0.49 \\
-\end{array}$ & 0.038427 & 0.13401 & 13 \\
\hline Dutch & 74 & - & 0.18 & - & - & - & - & - & & & 33 \\
\hline German (Caucasian) & 300 & 0.37 & 0.15 & 0.35 & 0.38 & - & 0.0 & - & 0.002137 & 0.078291 & 36 \\
\hline Israeli & 133 & 0.46 & 0.20 & 0.56 & 0.45 & 0.0 & 0.0 & 0.55 & 0.322641 & 0.571025 & 11 \\
\hline Japanese & \begin{tabular}{|c|}
120 \\
267 \\
27 \\
\end{tabular} & $\begin{array}{l}0.63 \\
0.64 \\
0.74 \\
\end{array}$ & $\begin{array}{l}0.16 \\
0.11 \\
0.19 \\
\end{array}$ & $\begin{array}{c}0.36 \\
- \\
0.26 \\
\end{array}$ & $\begin{array}{c}0.43 \\
- \\
0.26 \\
\end{array}$ & $\begin{array}{c}- \\
- \\
0.0\end{array}$ & $\begin{array}{l}- \\
- \\
0.0\end{array}$ & $\begin{array}{c}- \\
- \\
0.19\end{array}$ & $\begin{array}{c}<0.00001 \\
0.011239 \\
0.001111\end{array}$ & $\begin{array}{l}0.005088 \\
0.195709 \\
0.003401 \\
\end{array}$ & $\begin{array}{l}34 \\
41 \\
11 \\
\end{array}$ \\
\hline Korean & 24 & 0.75 & 0.25 & - & - & - & - & - & 0.775142 & 1.00000 & 35 \\
\hline Malaysian & $\begin{array}{r}100 \\
35\end{array}$ & $\begin{array}{l}0.87 \\
0.83\end{array}$ & $\begin{array}{l}0.11 \\
0.13\end{array}$ & $\begin{array}{c}0.24 \\
-\end{array}$ & $\begin{array}{c}0.50 \\
-\end{array}$ & $\begin{array}{c}0.0 \\
-\end{array}$ & - & - & $\begin{array}{c}<0.00001 \\
0.186616\end{array}$ & $\begin{array}{l}<0.00001 \\
0.403354\end{array}$ & $\begin{array}{l}25 \\
24\end{array}$ \\
\hline Pakistani & 192 & 0.47 & 0.09 & 0.56 & 0.26 & 0.0 & 0.005 & 0.59 & 0.008402 & 0.81518 & 11 \\
\hline Tanzanian & 366 & 0.87 & 0.06 & - & - & - & - & - & $<0.00001$ & 0.289424 & 38 \\
\hline Turkish & 94 & 0.46 & 0.12 & 0.0 .38 & 0.36 & - & 0.0 & - & 0.89754 & 0.289424 & 36 \\
\hline Macedonian & 266 & 0.41 & 0.14 & 0.65 & 0.40 & 0.0 & 0.0 & 0.47 & $0.928464^{\mathrm{d}}$ & & $\begin{array}{l}\text { this } \\
\text { study }\end{array}$ \\
\hline Albanian & 94 & 0.42 & 0.12 & 0.62 & 0.45 & 0.0 & 0.0 & 0.50 & 0.928464 & & $\begin{array}{c}\text { this } \\
\text { study }\end{array}$ \\
\hline Greek & 403 & 0.43 & 0.16 & - & - & - & - & - & 0.811389 & 0.595368 & 27 \\
\hline Caucasian & 423 & 0.37 & 0.15 & - & - & - & - & - & 0.472334 & 0.333625 & 32 \\
\hline
\end{tabular}

${ }^{a} n$ : number of patients.

b The $p$ value of differences in allele frequencies between Macedonians and different ethnic groups.

c The $p$ value of differences in allele frequencies between Albanians and different ethnic groups.

d The $p$ value of differences in allele frequencies between Albanians and Macedonians. 
Table 5. Alignment and frequencies of the SLCO1B1 haplotypes in 266 Macedonian subjects.

\begin{tabular}{|c|c|c|c|c|c|c|c|c|c|c|}
\hline SLCO1B1 Haplotype & $\begin{array}{l}\mathrm{c} .388 \\
\mathrm{~A}>\mathrm{G}\end{array}$ & \begin{tabular}{|l|}
$\mathrm{c} .521$ \\
$\mathrm{~T}>\mathrm{C}$
\end{tabular} & \begin{tabular}{|l|}
$\mathrm{c.571}$ \\
$\mathrm{T}>\mathrm{C}$
\end{tabular} & \begin{tabular}{|l|}
$c .597$ \\
$C>T$
\end{tabular} & $\begin{array}{l}c .1086 \\
C>T\end{array}$ & \begin{tabular}{|l|}
$c .1463$ \\
$\mathrm{G}>\mathrm{C}$
\end{tabular} & $\begin{array}{l}\text { c.*439 } \\
T>G\end{array}$ & \multicolumn{3}{|c|}{ Haplotypes Found } \\
\hline Reference & A & $\mathrm{T}$ & $T$ & $\mathrm{C}$ & C & G & $T$ & $n$ & $\%$ & $95 \% \mathrm{CI}$ \\
\hline$* 1 J / * 1 K / * 1 L^{a}$ & & & $\mathrm{C}$ & & & & & 148 & 39.1 & $0.747-1.338$ \\
\hline$* 18^{b} / \mathrm{New}^{\mathrm{c}}$ & G & & $\mathrm{C}$ & $T$ & & & G & 44 & 11.6 & $0.641-1.559$ \\
\hline$* 15^{a} / * 16^{d} / * 17^{c}$ & G & $\mathrm{C}$ & & $\mathrm{T}$ & & & G & 32 & 8.6 & $0.602-1.662$ \\
\hline$* 1 \mathrm{~J} /{ }^{*} 1 K^{*} 1 L^{a}$ & & & $\mathrm{C}$ & & & & G & 32 & 8.4 & $0.599-1.668$ \\
\hline$* 1 G / * 1 H^{a} / \mathrm{New}^{\mathrm{f}} / \mathrm{New}^{\mathrm{g}}$ & G & & & $T$ & & & G & 29 & 7.7 & $0.587-1.705$ \\
\hline$* 1 A^{a} / N_{e w}^{f} / * 1 E^{h}$ & & & & & & & & 22 & 5.9 & $0.548-1.25$ \\
\hline$* 1 B / * 1 F^{a} / \mathrm{New}^{\mathrm{f}}$ & G & & & & & & G & 11 & 3.1 & $0.441-2.265$ \\
\hline$* 20^{i} / * 21^{j} / \mathrm{New}^{\mathrm{f}}$ & G & & & $\mathrm{T}$ & & & & 11 & 3.0 & $0.432-2.315$ \\
\hline New & & & & $T$ & & & G & 8 & 2.1 & $0.375-2.667$ \\
\hline$* 5^{a} / \mathrm{New}^{\mathrm{f}}$ & & $\mathrm{C}$ & & & & & G & 7 & 2.0 & $0.360-2.779$ \\
\hline New & G & & C & & & & & 7 & 1.9 & $0.353-2.836$ \\
\hline$* 18^{k} / \mathrm{New}^{1}$ & G & & C & $\mathrm{T}$ & & & & 6 & 1.7 & $0.330-3.034$ \\
\hline New & G & C & & $T$ & & & & 5 & 1.5 & $0.308-3.250$ \\
\hline New & & & $\mathrm{C}$ & $T$ & & & G & 4 & 1.1 & $0.249-4.011$ \\
\hline $\mathrm{New}^{\mathrm{m}}$ & G & & C & & & & G & 2 & 0.6 & $0.167-6.006$ \\
\hline New & & & & & & & G & 1 & 0.5 & $0.130-7.712$ \\
\hline New & G & & C & & & & G & 1 & 0.3 & $0.082-12.232$ \\
\hline New & & & & $\mathrm{T}$ & & & & 1 & 0.3 & $0.073-13.647$ \\
\hline New & & C & & $T$ & & & & 1 & 0.3 & $0.068-14.662$ \\
\hline New & G & C & & & & & G & 1 & 0.3 & $0.064-15.593$ \\
\hline
\end{tabular}

95\% CI: 95\% confidence interval.

a The name includes the presented sequence of the SNPs investigated in this study and referent alleles of the additional SNPs investigated in the study by Pasanen et al. [13] (at positions g.-11187, g.-11110, g.-10499, c.411, c.463 and c.1929).

b,d,e, ,h,i,j,k The haplotype name includes a sequence of the SNPs investigated in this study and referent alleles in other SNPs investigated in the cited study [13], except at the following positions b: c.411 and c.463; d: g.-10499; e: g.-11187; h: g.-11110; i: c.1929; j: g.-11187 and c.1929, and k: c. 411 and c. 463 , where the variant alleles exist.

c,f,g,l The haplotype is assigned as new by Pasanen et al. [13], having the same sequence of the SNPs investigated in this study and referent alleles at other SNPs investigated in the cited study [13], except at the following positions c: g.-11110, c.411 and c.463; f: g.-11187; g: g.-10499, and I: c. 411, c. 463 and c. 1929 , where variant alleles exist.

${ }^{m}$ The haplotype is assigned as new by Pasanen et al. [13], having the same sequence of the SNPs investigated in this study and referent alleles in additional SNPs investigated in the cited study.

and c.521T $>$ C, were present in four haplotypes, of which the dominant haplotype $* 15 / * 16 / * 17$ had a frequency of $8.6 \%$.

In the Albanian population, 10 haplotypes occurred at a frequency equal or greater than $3.0 \%$ (Table 6). The most common haplotype was the same as in the Macedonian ethnic group, ${ }^{*} 1 J / * 1 K / * 1 L$, with a frequency of $26.6 \%$. The variant allele $\mathrm{C}$ at position c.571 existed in nine haplotypes with a frequency between 1.4 and $26.6 \%$. The variant allele $\mathrm{G}$ at position c.388 existed in 10 haplotypes, while c.597C $>$ T SNP in 11 haplotypes, both occurring at frequencies between 1.0 and $12.4 \%$. The c. ${ }^{*} 439 \mathrm{~T}>\mathrm{G}$ occurred in 10 haplotypes, with a frequency between 0.6 and $12.4 \%$, and the c.521T $>$ C SNP existed in seven haplotypes, with a frequency between 0.6 and $3.7 \%$. Three of the haplotypes contained the variant alleles of the c.388A $>\mathrm{G}$ and c.521 $\mathrm{T}>\mathrm{C}$ SNPs with a frequency $\geq 1.0 \%$, with the major haplotype $* 15 / * 16 / * 17$ having a frequency of $2.4 \%$.

\section{DISCUSSION}

It is clearly evident that the mutations in the $S L$ $C O 1 B 1$ gene and their clinical significance for a large number of endogenous and xenobiotic substrates 
Table 6. Alignment and frequencies of the SLCO1B1 haplotypes in 94 Albanian subjects.

\begin{tabular}{|c|c|c|c|c|c|c|c|c|c|c|}
\hline SLCO1B1 Haplotype & \begin{tabular}{|l|}
$\mathrm{c} .388$ \\
$\mathrm{~A}>\mathrm{G}$
\end{tabular} & \begin{tabular}{|l|}
$\mathrm{c.521}$ \\
$\mathrm{T}>\mathrm{C}$
\end{tabular} & \begin{tabular}{|l|} 
c.571 \\
T $>C$
\end{tabular} & \begin{tabular}{|l|} 
c.597 \\
C>T
\end{tabular} & $\begin{array}{l}c .1086 \\
C>T\end{array}$ & \begin{tabular}{|l}
$c .1463$ \\
$G>C$
\end{tabular} & $\begin{array}{l}\text { c. } * 439 \\
T>G\end{array}$ & \multicolumn{3}{|c|}{ Haplotypes Found } \\
\hline Reference & A & $\mathbf{T}$ & $\mathbf{T}$ & C & C & G & $\mathbf{T}$ & $n$ & $\%$ & $95 \% \mathrm{CI}$ \\
\hline$* 1 J / * 1 K / * 1 L^{a}$ & & & C & & & & & 45 & 26.6 & $0.618-1.618$ \\
\hline$* 18^{b} \mathrm{New}^{\mathrm{c}}$ & G & & C & $\mathrm{T}$ & & & G & 21 & 12.4 & $0.525-1.905$ \\
\hline$* 1 G / * 1 H^{a} / \mathrm{New}^{\mathrm{f}} / \mathrm{New}^{\mathrm{g}}$ & G & & & $\mathrm{T}$ & & & G & 19 & 11.5 & $0.514-1.947$ \\
\hline$* 1 J / * 1 K / * 1 L^{a}$ & & & C & & & & G & 11 & 6.7 & $0.424-3.344$ \\
\hline$* 1 A^{a} / * 1 E^{h} / \mathrm{New}^{\mathrm{f}}$ & & & & & & & & 8 & 5.1 & $0.380-2.630$ \\
\hline$* 1 \mathrm{~B} /{ }^{*} 1 \mathrm{~F}^{\mathrm{a}} / \mathrm{New}^{\mathrm{f}}$ & G & & & & & & G & 6 & 3.8 & $0.330-0.301$ \\
\hline$* 5^{\mathrm{a}} / \mathrm{New}^{\mathrm{f}}$ & & C & & $\mathrm{T}$ & & & G & 6 & 3.7 & $0.322-3.103$ \\
\hline New & & & C & $\mathrm{T}$ & & & G & 6 & 3.5 & $0.316-3.162$ \\
\hline New & & & & & & & G & 5 & 3.2 & $0.297-3.370$ \\
\hline New & G & & C & & & & & 5 & 3.0 & $0.286-3.497$ \\
\hline New & & C & C & $\mathrm{T}$ & & & & 4 & 2.9 & $0.280-3.573$ \\
\hline$* 20^{i} / * 21^{j} / \mathrm{New}^{\mathrm{f}}$ & G & & & $\mathrm{T}$ & & & & 4 & 2.7 & $0.268-3.734$ \\
\hline$* 1 \mathrm{~B} / * 1 \mathrm{~F}$ & G & & & . & & & & 4 & 2.5 & $0.259-3.865$ \\
\hline$* 15^{a} / * 16^{k} / * 17^{l}$ & G & C & & $\mathrm{T}$ & & & G & 4 & 2.4 & 0.251-3.990 \\
\hline New & & & & $\mathrm{T}$ & & & & 3 & 2.2 & $0.236-4.236$ \\
\hline $\mathrm{New}^{\mathrm{m}}$ & G & & C & . & & & G & 3 & 1.9 & $0.212-4.716$ \\
\hline New & & & C & $T$ & & & & 3 & 1.9 & $0.210-4.755$ \\
\hline New & G & C & C & $\mathrm{T}$ & & & & 2 & 1.4 & $0.168-5.938$ \\
\hline New & G & C & & $\mathrm{T}$ & & & & 1 & 1.0 & 0.114-8.806 \\
\hline New & & $\mathrm{C}$ & & & & & & 1 & \begin{tabular}{|l|}
0.7 \\
\end{tabular} & $0.082-12.179$ \\
\hline $\mathrm{New}^{\mathrm{m}}$ & & C & & & & & G & 1 & 0.6 & $0.065-15.451$ \\
\hline
\end{tabular}

95\% CI: 95\% confidence interval.

a The name includes the presented sequence of the SNPs investigated in this study and referent alleles of the additional SNPs investigated in the study by Pasanen et al. [13].

$\mathbf{b}, \mathbf{h}, \mathbf{i , j}, \mathbf{j}, \mathbf{l}, \mathbf{l}$ The haplotype name includes a sequence of the SNPs investigated in this study and referent alleles in other SNPs investigated in the cited study [13], except at the following positions b: c.411 and c.463; h: g.-11110; i: c.1929; j: g.-11187 and c.1929; k: g.-10499; and l: g.-11187, where variant alleles exist.

c,f,g The haplotype is assigned as new by Pasanen et al. [13], having the same sequence of the SNPs investigated in this study and referent alleles in other SNPs investigated in the cited study, except at the following positions c: g.-11110, c.411 and c.463; f: g.-11187; and g: g.-10499, where variant alleles exist.

${ }^{\mathrm{m}}$ The haplotype is assigned as new by Pasanen et al. [13], having the same sequence of the SNPs investigated in this study and referent alleles at additional SNPs investigated in the cited study (at positions g.-11187, g.-11110, g.-10499, c.411, c.463 and c.1929).

transported by OATP1B1 is a persistent motivation for scientific research. To the best of our knowledge, this is the first study in which polymorphisms contained in the SLCOIB1 gene were studied in the populations living in the Western Balkan Peninsula. For this reason, commonly seen mutations (c.388A $>\mathrm{G}$, c. $521 \mathrm{~T}>\mathrm{C}$, c. $571 \mathrm{~T}>\mathrm{C}$, c. $597 \mathrm{C}>\mathrm{T}, \mathrm{c} . * 439 \mathrm{~T}>\mathrm{G})$ as well as coding region SNPs that were not identified in the Caucasian (European) population (c.1086C $>\mathrm{T}$, c. $1463 \mathrm{G}>\mathrm{C}$ ) were selected for genotyping. Our data confirmed that SLCO1B1 is highly polymorphic and that several variants appear at a high frequency, both in the Macedonian and Albanian populations. The SNPs c.388A $>\mathrm{G}$ (Asn130Asp), c.571T $>\mathrm{C}$ (Leu191Leu), c.597C $>$ T (Phe199 Phe) and c. ${ }^{* 439 T}>\mathrm{G}$, all occurred with an allelic frequency between 40.0 and $65.0 \%$. The non synonymous c.521T $>$ C SNP, which has been constantly associated with a reduced OATP1B1 activity, was found with an allele frequency of approximately 14.0 and $12.0 \%$ in the Macedonian and Albanian population, respectively, which is nearly equal to that reported for Caucasians (15.0\%) [32], slightly lower than that reported previously for Dutch (18.0\%) [33], Finish (20.0\%) [13], 


\begin{tabular}{|l|c|c|c|c|c|c|c|}
\hline & $\mathbf{c . 3 8 8 A}>\mathbf{G}$ & $\mathbf{c . 5 2 1 T}>\mathbf{C}$ & $\mathbf{c . 5 7 1 T}>\mathbf{C}$ & $\mathbf{c . 5 9 7 C}>\mathbf{T}$ & $\mathbf{c . 1 0 8 6 C}>\mathbf{T}$ & $\mathbf{c . 1 4 6 3 G}>\mathbf{C}$ & $\mathbf{c}$ *439T>G \\
\hline c.388A $>$ G & & 0.698 & 0.487 & 0.740 & 0.000 & 0.000 & 0.613 \\
\hline $\mathbf{c . 5 2 1 T}>\mathbf{C}$ & 0.113 & & 0.919 & 0.872 & 0.000 & 0.000 & 0.645 \\
\hline c.571T $>$ C & 0.187 & 0.233 & & 0.520 & 0.000 & 0.000 & 0.426 \\
\hline c.597C $>$ T & 0.531 & 0.178 & 0.218 & & 0.000 & 0.000 & 0.699 \\
\hline c.1086C $>$ T & 0.000 & 0.000 & 0.000 & 0.000 & & 0.000 & 0.000 \\
\hline c.1463G $>$ C & 0.000 & 0.000 & 0.000 & 0.000 & 0.000 & & 0.000 \\
\hline c. *439T>G & 0.289 & 0.072 & 0.115 & 0.373 & 0.000 & 0.000 & \\
\hline
\end{tabular}

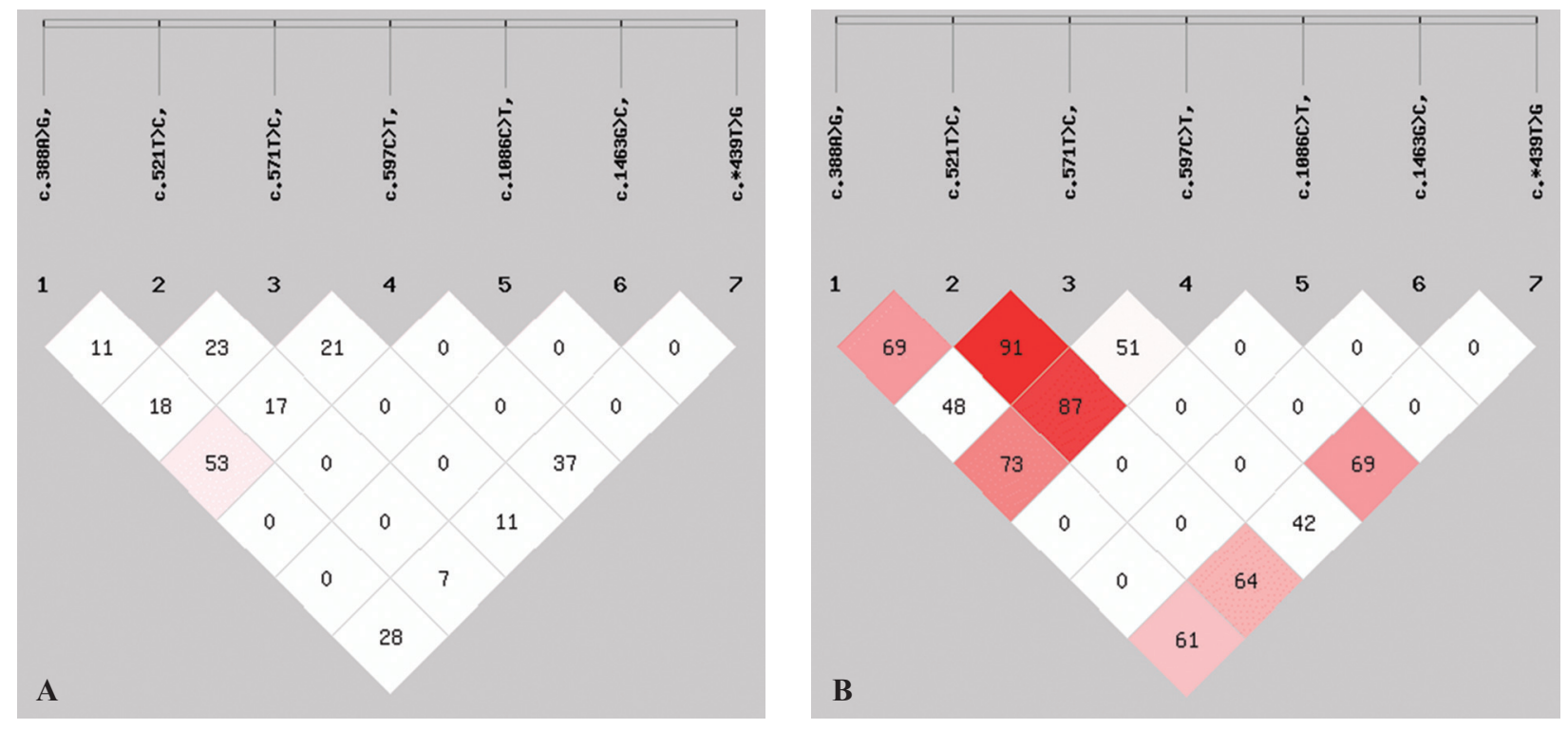

Figure 1. Pairwise LD profiles for SLCO1B1 SNPs in Macedonians ( $\mathrm{n}=266)$; $\mathrm{r} 2$ cells (below the diagonal, A) and D' (cells above the diagonal, B) values for each pair of the seven SNPs are presented.

Algerian (17.0\%) [11], Israeli (20.0\%) [11], Japanese (16.0-19.0\%) [11,34] and Korean (25.0\%) [35] ethnic groups, and much higher than that reported for African Americans (2.3\%) [12] and Sub-Saharan Africans (1.9\%) [11] (Table 4). So far, literature data point to equal allele frequency for this SNP in Macedonians and European Americans [12] and Han Chinese [29], although a lower number of subjects in the last two groups was included in the study. The same was observed in Albanian and Turkish subjects, with an equal number of subjects in the study [36]. Compared to studies with Native Americans, Caucasian Europeans, Sub-Saharan Africans, Japanese and Israeli subjects [11], the variant alleles found in the Macedonian and Albanian subjects were lower for c. $388 \mathrm{~A}>\mathrm{G}(41.0-42.0 \%$ vs. 46.0-79.0\%), higher for c. $571 \mathrm{~T}>\mathrm{C}(62.0-65.0 \%$ vs. $13.0-61.0 \%)$ and nearly equal for c. $597 \mathrm{C}>\mathrm{T}(40.0-45.0 \%$ vs. $42.0-50.0 \%)$, with the exception of Native Americans in which a much lower allele frequency was observed (28.0\%). The allele frequency for SNP c.*439T $>\mathrm{G}$ was lower in comparison with Sub-Saharan Africans (47.0-50.0\% vs. $76.0 \%$ ), higher than that of Caucasian Europeans $(30.0 \%)$ and almost equal to the frequency of other ethnic groups, where a variant $G$ allele existed with a frequency between $41.0 \%$ (Native Americans) and $55.0 \%$ (Israeli) (Table 4). No variant alleles were found for c. $1086 \mathrm{C}>\mathrm{T}$ and c. $1463 \mathrm{G}>\mathrm{C}$ SNPs in the Macedonian and Albanian populations and the same was observed in German, Finish, Japanese, Israeli and Turkish subjects $[11,36]$, while in the studied Native American and Sub-Saharan African, Ugandan and Pakistani ethnic groups, a low frequency of variant alleles was observed, between 1.0 and $7.0 \%$ for vari- 


\begin{tabular}{|l|c|c|c|c|c|c|c|}
\hline & $\mathbf{c . 3 8 8 A}>\mathbf{G}$ & $\mathbf{c . 5 2 1 T}>\mathbf{C}$ & $\mathbf{c . 5 7 1 T}>\mathbf{C}$ & $\mathbf{c . 5 9 7 C}>\mathbf{T}$ & $\mathbf{c . 1 0 8 6 C}>\mathbf{T}$ & $\mathbf{c . 1 4 6 3 G}>\mathbf{C}$ & $\mathbf{c}$ *439T $>\mathbf{G}$ \\
\hline $\mathbf{c . 3 8 8 A}>\mathbf{G}$ & & 0.219 & 0.370 & 0.498 & 0.000 & 0.000 & 0.477 \\
\hline $\mathbf{c . 5 2 1 T}>\mathbf{C}$ & 0.009 & & 0.635 & 0.746 & 0.000 & 0.000 & 0.238 \\
\hline $\mathbf{c . 5 7 1 T}>\mathbf{C}$ & 0.119 & 0.091 & & 0.206 & 0.000 & 0.000 & 0.137 \\
\hline $\mathbf{c . 5 9 7 C}>\mathbf{T}$ & 0.221 & 0.097 & 0.031 & & 0.000 & 0.000 & 0.505 \\
\hline c.1086C $>$ T & 0.000 & 0.000 & 0.000 & 0.000 & & 0.000 & 0.000 \\
\hline c.1463G $>$ C & 0.000 & 0.000 & 0.000 & 0.000 & 0.000 & & 0.000 \\
\hline c.*439T $>$ G & 0.169 & 0.008 & 0.011 & 0.214 & 0.000 & 0.000 & \\
\hline
\end{tabular}
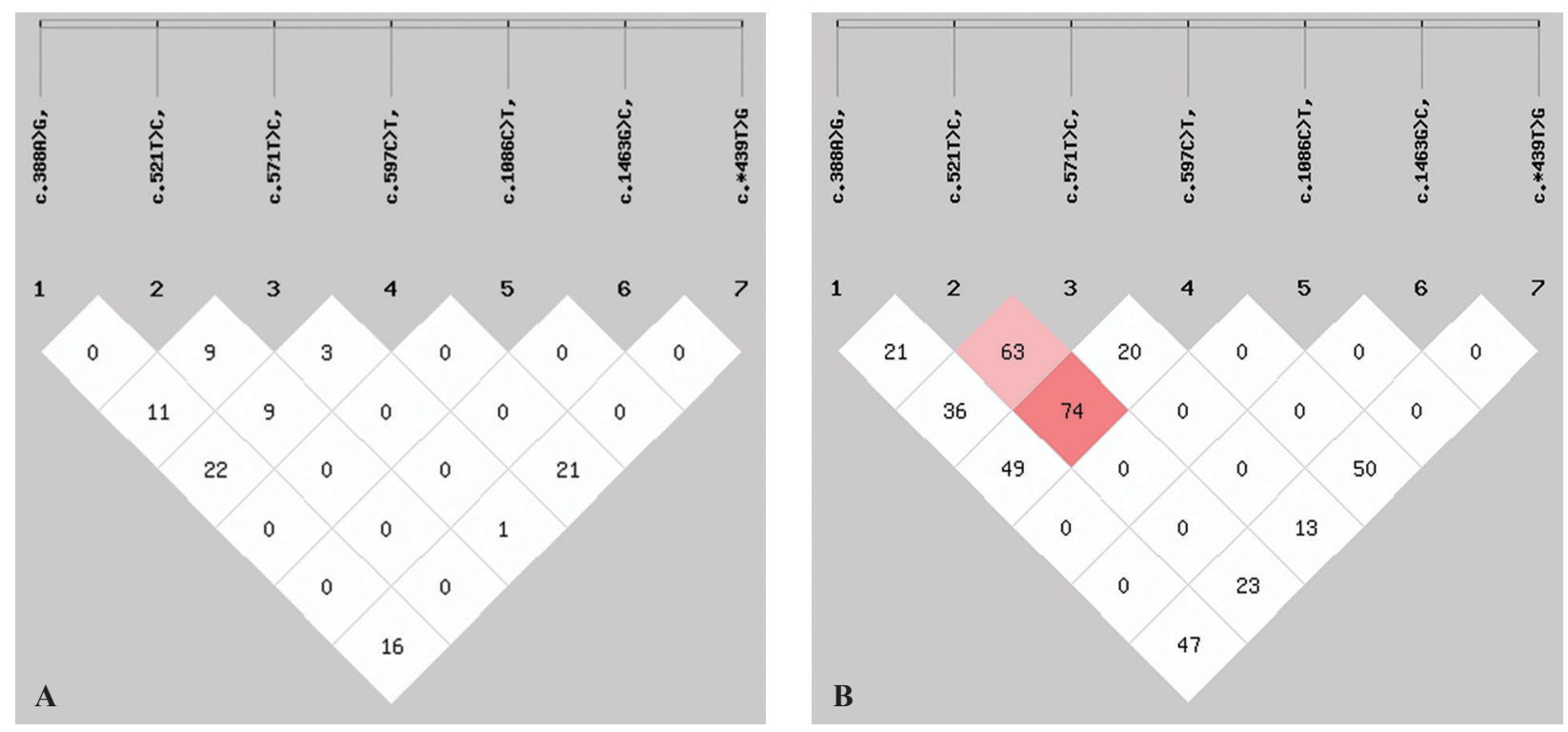

Figure 2. Pairwise LD profiles for SLCO1B1 SNPs in Albanians ( $=94) ; \mathrm{r} 2$ cells (below the diagonal, A) and D' (cells above the diagonal, B) values for each pair of the seven SNPs are presented.

ant $\mathrm{T}$ in c. $1086 \mathrm{C}>\mathrm{T}$ and 0.5 and $3.0 \%$ for variant $\mathrm{C}$ in c. $1463 \mathrm{G}>\mathrm{C}$ [11].

For all SNPs, the distributions of the genotypes did not differ significantly ( $p>0.05)$ between healthy subjects and patients and between male and female subjects. These data are partly in accordance with the results obtained in the study of Hubacek et al. [37], in which no difference for genotype distributions of rs4149056 variant between male and female subjects was observed. However, the results of the same study pointed to possible gender-dependent effects of this SNP within the SLCO1B1 gene on statin treatment efficacy.

It is increasingly evident that the most relevant variants, the SNPs $388 \mathrm{~A}>\mathrm{G}$ and $521 \mathrm{C}>\mathrm{T}$, have a major effect on OATP1B1 activity. However, their association and other SNPs in LD with these func- tionalities may modify the respective phenotype and explain the discrepant effects of some SNPs on OAT$\mathrm{P} 1 \mathrm{~B} 1$ activity in vivo. Most of the literature data point to a strong association between this SNP pair and its effect on drug response [20,29]. In the actual study, the association between c.388A $>\mathrm{G}$ and c.521 $\mathrm{T}>\mathrm{C}$ was relatively weaker compared to other SNP pairs, especially those in the Albanian population. These data and generally, the differences between the two populations in LD data, are probably explained by the significantly smaller number of Albanian subjects included in the study and random sampling variation. The c.521T $>$ C SNP showed the strongest correlation with the c. $597 \mathrm{C}>\mathrm{T}$ in both populations and the similar results have been obtained in the study of Pasanen et al. [13], in which a large sample (468) of Caucasian subjects was included. 
Compared with the analysis performed with single SNPs, haplotypes often better predict the associated phenotype. In the present study, the most common SLCO1B1 haplotype, *IJ/*IK/*IL, contained the synonymous c.571 $\mathrm{T}>\mathrm{C}$ SNP as compared with the reference sequence. It occurred at a frequency $(35.6 \%)$ similar to that reported in the study of Pasanen et al. [13]. The c.521T $>$ C SNP existed in two $(* 5$ and $* 15 / * 16 / * 17)$ major haplotypes in the Macedonian and Albanian populations and one new, identified in the Albanian population only. Both common haplotypes, with a frequency of 2.0 and $3.7 \%$ (for *5) and 2.4 and $8.6 \%$ (for *15/*16/*17) in the Macedonian and Albanian subjects, respectively, contained the c. $597 \mathrm{C}>\mathrm{T}$ and c.439T $>$ G SNPs. In the new haplotype identified in the Albanian population, instead of variant $G$ allele in the c.439T $>G$ SNP, variant $\mathrm{C}$ allele of c.571T $>C$ existed, with referent alleles in other SNPs. Considering the significantly smaller number of subjects in the Albanian population, as potential limitation of this study, this result should be confirmed in a study in which a larger number of Albanian subjects would be included. The frequencies of the major haplotype $* 15 / * 16 / * 17$ containing the variant alleles of the functionally most significant SNP pair c. $388 \mathrm{~A}>\mathrm{G} / \mathrm{c} .521 \mathrm{~T}>\mathrm{C}$ (8.6 and $2.4 \%$ for Macedonians and Albanians, respectively) were lower than the frequency of haplotype $* 15$ reported for Chinese (14.0\%) and Japanese (15.8\%), higher for Macedonians and comparable for Albanians with that of Caucasians $(2.4 \%)$ and significantly higher than the one of African Americans $(0.0 \%)[13,29]$.

In conclusion, this study presents an extensive analysis of SLCO1B1 variant genotype and haplotype distribution in selected populations living in the Western Balkan Peninsula, Macedonians and Albanians for the first time. No significant differences $(p>0.05)$ in allelic frequencies and genotype distributions of the analyzed SNPs were observed between the two ethnic groups and the data are similar to those for Caucasians. About 8.6 and $2.4 \%$ of the Macedonians and Albanians, respectively, carrying the $S L C O 1 B 1 * 15$ or SLCO1B $1 * 16$ or SLCO1B $1 * 17$ variant may exhibit altered/impaired transport activity of OATP1B1.

Declaration of Interest. The authors report no conflicts of interest. The authors alone are responsible for the content and writing of this article.

\section{REFERENCES}

1. Liu J, Long J, Zhang S, Fang X, Luo Y. Polymorphic variants of SLCO1B1 in neonatal hyperbilirubinemia in China. Ital J Pediatr. 2013; 39(49): 1-5.

2. Lopez-Lopez E, Martin-Guerrero I, Ballesteros J, Pican MA, Garcia-Miguel P, Navajas A, et al. Polymor- phisms of the SLCO1B1 gene predict methotrexate-related toxicity in childhood acute lymphoblastic leukemia. Pediatr Blood Cancer. 2011; 57(4): 612-619.

3. Niemi M, Kivistц KT, Hofmann U, Schwab M, Eichelbaum M, Fromm MF. Fexofenadine pharmacokin-etics is associated with a polymorphism of the SLCO1B1 gene (encoding OATP1B1). Br J Clin Pharmacol. 2005; 59(5): 602-604.

4. Niemi M, Backman JT, Kajosaari LI, Leathart JB, Neuvonen M, Daly AK, et al. Polymorphic organic anion transporting polypeptide $1 \mathrm{~B} 1$ is a major determinant of repaglinide pharmacokinetics. Clin Pharmacol Ther. 2005; 77(6): 468-478.

5. Pasanen MK, Fredrikson H, Neuvonen PJ, Niemi M. Different effects of SLCO1B1 polymorphism on the phar-macokinetics of atorvastatin and rosuvastatin. Clin Phar-macol Ther. 2007; 82(7): 726-733.

6. Ho RH, Tirona RG, Leake BF, Glaeser H, Lee W, Lemke CJ, et al. Drug and bile acid transporters in rosuva statin hepatic uptake: Function, expression and pharmaco genetics. Gastroenterology. 2006; 130(6): 1793-1806.

7. Yamada A, Maeda K, Kamiyama E, Sugiyama $\mathrm{D}$, Kondo T, Shiroyanagi Y, et al. Multiple human isoforms of drug transporters contribute to the hepatic and renal transport of olmesartan, a selective antagonist of the angiotensin II AT1receptor. Drug Metab Dispos. 2007; 35(12): 2166-2176.

8. Liu L, Cui Y, Chung AY, Shitara Y, Sugiyama Y, Keppler D, et al. Vectorial transport of enalapril by Oatp 1a1/Mrp2 and OATP1B1 and OATP1B3/ MRP2 in rat and human livers. J Pharmacol Exp Ther. 2006; 318(1): 395-402.

9. Neuvonen PJ, Niemi M, Backman JT. Drug interactions with lipid-lowering drugs: Mechanisms and clinical relevance. Clin Pharmacol Ther. 2006; 80(6): 565-581. 
10. Vavricka SR, Van Mootfort J, Ha HR, Meier PJ, Fattinger K. Interactions of ryfamycin Sv and rifampicin with organic anion uptake systems of human liver. Hepatology. 2002; 36(1): 164-172.

11. Pasanen MK, Neuvonen PJ, Niemi M. Global analysis of genetic variation in SLCO1B1. Pharmacogenomics. 2008; 9(1): 19-33.

12. Tirona RG, Leake BF, Merino G, Kim RB. Poly-morphisms in OATP-C: Identification of multiple allelic variants associated with altered transport activity among European- and AfricanAmericans. J Biol Chem. 2001; 276(38): 3566935675 .

13. Pasanen MK, Backman JT, Neuvonen PJ, Niemi M. Frequencies of single nucleotide polymorphisms and haplotypes of organic anion transporting polypeptide 1B1 SLCO1B1 gene in a Finnish population. Eur J Clin Pharmacol. 2006; 62(6): 409-415.

14. Maeda K, Ieiri I, Yasuda K, Fujino A, Fujiwara $\mathrm{H}$, Otsubo $\mathrm{K}$, et al. Effects of organic anion transporting polypeptide 1B1 haplotype on pharmacokinetics of pravastatin, valsartan and temocapril. Clin Pharmacol Ther. 2006; 79(5): 427-439.

15. Oswald S, Konig J, Lutjohann D, Giessmann T, Kroemer HK, Rimmbach C, et al. Disposition of ezetimibe is influenced by polymorphisms of the hepatic uptake carrier OATP1B1. Pharmacogenet Genomics. 2008; 18(7): 559-568.

16. Niemi M, Pasanen MK, Neuvonen PJ. SLCO1B1 polymoprhism and sex affect the pharmacokinetics of pra-vastatin but not fluvastatin. Clin Pharmacol Ther. 2006; 80(4): 356-366.

17. Han JY, Lim HS, Shin ES, Yoo YK, Park YH, Lee $\mathrm{JE}$, et al. Influence of the organic anion-transporting polypeptide 1B1 (OATP1B1) polymorphisms on irinotecan - Pharmacoknetics and clinical outcome of patients with advanced non-small lung cancer. Lung Cancer. 2008; 59(1): 69-75.

18. SEARCH Collaborative group, Link E, Parish S, Armitage J, Bowman L, Heath S, Matsuda $\mathrm{F}$, et al. SLCO $1 B 1$ variants and statin-induced myopathy - A genomewide study. N Engl J Med. 2008; 359(8): 789-799.

19. Santos PC, Gagliardi ACM, Miname MH, Chacra AP, Santos RD, Krieger JE, et al. SLCO1B1 haplotypes are not associated with atorvastatininduced myalgia in Brazilian patients with familial hypercholesterolemia. Eur J Clin Pharmacol. 2012; 68(3): 273-279.

20. Rodrigues AC, Perin PM, Purim SG, Silbiger VN, Genvigir FD, Willrich MA, et al. Pharmacogenetics of OATP transporters reveals that SLCO1B1 c.388A $>$ G variant is determinant of increased atorvastatin response. Int J Mol Sci. 2011; 12(9): 5815-5827.

21. Kameyama Y, Yamashita K, Kobayashi K, Hosokawa M, Chiba K. Functional characterization of SLCO 1B1 (OATP-C) variants, SLCO1B1 *5, SLCO1B $1 * 15$ and SLCO1B1 $* 15+\mathrm{C} 1007 \mathrm{G}$, by using transient expression systems of HeLa and HEK 293 cells. Pharmacogenet Genomics. 2005; 15(7): 513-522.

22. Niemi M, Schaeffeler E, Lang T, Fromm MF, Neuvonen M, Kyrklund C, et al. High plasma pravastatin concentrations are associated with single nucleotide polymorphisms and haplotypes of organic transporting polypep-tide-C (OATPC, SLCO1B1). Pharmacogenetics. 2004; 14(7): 429-440.

23. Couvert P, Giral P, Dejager S, Gu J, Huby T, Chapman MJ, et al. Association between a frequent allele of the gene encoding OATP1B1 and enhanced LDL-lowering response to fluvastatin therapy. Pharmacogenomics. 2008; 9(9): 12171227.

24. Lee E, Ryan S, Birmingham B, Zalikowski J, March R, Ambrose H, et al. Rosuvastatin pharmacokinetics and pharmacogenetics in white and Asian subjects residing in the same environment. Clin Pharmacol Ther. 2005; 78(4): 330-341.

25. Jada SR, Xiaochen S, Yan LY, Xiaoqiang X, Lal S, Zhou SF, et al. Pharmacogenetics of $S L$ CO1B1: Haplotypes, htSNPs and hepatic expression in three distinct Asian populations. Eur J Clin Pharmacol. 2007; 63(6): 555-563.

26. Nozawa T, Nakajima M, Tamai I, Noda K, Nezu J, Sai Y, et al. Genetic polymorphisms of human organic anion transporters OATP-C (SLC21A6) and OATP-B (SLC21A9): Allele frequencies in the Japanese population and functional analysis. J Pharmacol Exp Ther. 2002; 302(2): 804-813.

27. Giannakopoulou E, Ragia G, Kolovou V, Tavri$\operatorname{dou}$ A, Tselepis AD, Elisaf M, et al. No impact 
of SLCO1B1 521T>C, 388A $>$ G and $411 \mathrm{G}>\mathrm{A}$ polymorphisms on response to statin therapy in the Greek population. Mol Biol Rep. 2014; 41(7): 4631-4638.

28. Santos PC, Soares RA, Nascimento RM, Machado-Coelho GLL, Mill JG, Krieger JE, et al. SLCO1B1 rs414 9056 polymorphism associated with statin-induced myopathy is differently distributed according to ethnicity in the Brazilian general population: Amerindians as a high risk ethnic group. BMC Med Genet. 2011; 12(136): 1-6.

29. Xu LY, He YJ, Zhang W, Deng S, Li Q, Zhang $\mathrm{WX}$, et al. Organic anion transporting polypeptide-1B1 haplotypes in Chinese patients. Acta Pharmacologica Sinica. 2007; 28(10): 1693-1697.

30. Daka A, Nestorovska AK, Radivojsa I, Mladenovska K, Vavlukis M, Dimovski A. Frequency of organic anion transporting polypeptide 1B1 SLCO1B1 gene variants in populations of patients treated with atorvastatin. Proceedings of the 41st European Society of Clinical Pharmacy Symposium on Clinical Pharmacy, October 2931 2012, Barcelona, Spain.

31. Shi YY, He L. SHEsis, a powerful software platform for analyses of linkage disequilibrium, haplotype construction, and genetic association at polymorphism loci. Cell Res. 2005; 15(2): 97-98.

32. Mwinyi J, Johne A, Bauer S, Roots I, Gerloff T. Evidence for inverse effects of OATP-C (SLC21A6) 5 and $1 \mathrm{~b}$ haplotypes on pravastatin kinetics. Clin Pharmacol Ther. 2004; 75(5): 415-421.

33. Brunham LR, Lansberg PJ, Zhang L, Miao F, Carter C, Hovingh GK, et al. Differential effect of the rs4149056 variant in SLCO1B1 on myopathy associated with simvastatin and atorvastatin. Pharmacogenomics J. 2012; 12(3): 233-237.
34. Nishizato Y, Ieiri I, Suzuki H, Kimura M, Kawabata K, Hirota T, et al. Polymorphisms of OATPC (SLC 21A6) and OAT3 (SLC22A8) genes: Consequences for pravastatin pharmacokinetics. Clin Pharmacol Ther. 2003; 73(6): 554-565.

35. Chung JY, Cho JY, Yu KS, Kim JR, Oh DS, Jung $\mathrm{HR}$, et al. Effect of OATP1B1 (SLCO1B1) variant alleles on the pharmacokinetics of pitavastatin in healthy volunteers. Clin Pharmacol Ther. 2005; 78(4): 342-350.

36. Mwinyi J, Kopke K, Schaefer M, Roots I, Gerloff T. Comparison of SLCOIB1 sequence variability among German, Turkish, and African populations. Eur J Clin Pharmacol. 2008; 64(3): 257266.

37. Hubacek JA, Dlouha D, Adamkova V, Lanska V, Ceska R, Vrablik M. Possible gene-gender interaction between the SLCO1B1 polymorphism and statin treatment efficacy. Neuro Endocrinol Lett. 2012; 33(Suppl 2): 22-25.

38. Aklillu E, Mugusi S, Ngaimisi E, Hoffmann MM, Kцnig S, Ziesenitz V, et al. Frequency of the SLCO1B1 388A $>\mathrm{G}$ and the 521T $>\mathrm{C}$ polymorphism in Tanzania genotyped by a new LightCycler®-based method. Eur J Clin Pharmacol. 2011; 67(11): 1139-1145.

39. Yang GP, Yuan H, Tang B, Zhang W, Wang LS, Huang ZJ, et al. Lack of effect of genetic polymorphisms of SLCO1B1 on the lipid-lowering response to pitavastatin in Chinese patients. Acta Pharmacologica Sinica. 2010; 31(3): 382-386.

40. Lin R, Wang X, Zhou W, Fu W, Wang Y, Huang $\mathrm{W}$, et al. Association of polymorphisms in the solute carrier organic anion transporter family member 1B1 gene with essential hypertension in the Uyghur population. Ann Hum Genet. 2011; 75(2): 305-311. 
